\title{
1 Single-cell RNA sequencing of Plasmodium vivax sporozoites reveals 2 stage- and species-specific transcriptomic signatures
}

3 Anthony A. Ruberto ${ }^{1, \ddagger, \alpha}$, Caitlin Bourke ${ }^{2,3, \ddagger}$, Amélie Vantaux $^{4}$ Steven P. Maher ${ }^{5}$, Aaron Jex $^{2,3}$,

4 Benoit Witkowski ${ }^{4}$, Georges Snounou ${ }^{6}$, Ivo Mueller ${ }^{1,2,3 *}$

5

$6 \quad{ }^{1}$ Department of Parasites and Insect Vectors, Institut Pasteur, Paris, France

$7 \quad{ }^{2}$ Division of Population Health and Immunity, Walter and Eliza Hall Institute of Medical

8 Research, Parkville, Victoria, Australia

$9{ }^{4}$ Malaria Molecular Epidemiology Unit, Institut Pasteur du Cambodge, Phnom Penh, Kingdom

10 of Cambodia

$11{ }^{3}$ Department of Medical Biology, The University of Melbourne, Parkville, Victoria Australia

$12{ }^{5}$ Center for Tropical and Emerging Global Diseases, University of Georgia, Athens 30602, USA

$13{ }^{6}$ Commissariat à l'Énergie Atomique et aux Énergies Alternatives-Université Paris Sud 11-

14 INSERM U1184, Immunology of Viral Infections and Autoimmune Diseases (IMVA-HB),

15 Infectious Disease Models and Innovative Therapies (IDMIT) Department, Institut de Biologie

16 François Jacob (IBFJ), Direction de la Recherche Fondamentale (DRF), Fontenay-aux-Roses,

17 France

${ }^{\ddagger} \mathrm{AAR}$ and $\mathrm{CB}$ contributed equally to this work.

$20{ }^{\circledR}$ Current address: Center for Tropical and Emerging Global Diseases, University of Georgia,

21 Athens 30602, USA

22

23

* Corresponding author

24

25 E-mail: mueller@wehi.edu.au 


\section{Abstract}

27 Plasmodium vivax sporozoites reside in the salivary glands of a mosquito before infecting a

28 human host. Previous transcriptome-wide studies in populations of these forms were limited in

29 their ability to elucidate cell-to-cell variation, thereby masking cellular states potentially important

30 in understanding transmission outcomes. In this study, we performed transcription profiling on

319,947 P. vivax sporozoites to assess the extent to which they differ at single-cell resolution. We

32 show that sporozoites residing in the mosquito's salivary glands exist in distinct developmental

33 states, as defined by their transcriptomic signatures. Additionally, relative to $P$. falciparum, $P$.

34 vivax displays overlapping and unique gene usage patterns, highlighting conserved and species-specific gene programs. Notably, distinguishing $P$. vivax from $P$. falciparum were a subset of $P$. vivax sporozoites expressing genes associated with translational regulation and repression. Finally, our comparison of single-cell transcriptomic data from $P$. vivax sporozoite and erythrocytic forms reveals gene usage patterns unique to sporozoites. In defining the transcriptomic signatures of individual $P$. vivax sporozoites, our work provides new insights into the factors driving their developmental trajectory and lays the groundwork for a more comprehensive $P$. vivax cell atlas. 


\section{Introduction}

Malaria remains the most significant parasitic disease of humans globally, causing an estimated 229 million infections and 409,000 deaths per year (World Health Organization, 2020). Plasmodium spp. are the etiological agents of malaria, and at least five species are known to infect humans (World Health Organization, 2020). Plasmodium falciparum and $P$. vivax are the most prevalent, and both contribute significantly to disease burden (Battle and Baird, 2021; Battle et al., 2019; Weiss et al., 2019). Plasmodium spp. infection in humans begins with the deposition of sporozoites into the dermis when an infected Anopheles mosquito takes a blood meal (Amino et al., 2006). While sporozoites must undergo replication in the liver before mounting a blood-stage infection, $P$. vivax sporozoites can develop into either a replicating or persisting (hypnozoite) form (Krotoski, 1985). Hypnozoites can remain in the liver for weeks, months or years before reactivating to undergo schizogony (Krotoski et al., 1982), leading to a relapsing blood-stage infection. Relapsing infections are estimated to comprise up to $90 \%$ of $P$. vivax malaria cases in some regions (Adekunle et al., 2015; Commons et al., 2020; Robinson et al., 2015). Relapse-causing hypnozoites, in addition to a high prevalence of subdetectable and often asymptomatic blood-stage infections, severely limit efforts to eradicate $P$. vivax malaria (Battle et al., 2019; Sattabongkot et al., 2018). Recent modelling suggests that eliminating $P$. vivax malaria is not possible without programs that specifically target and cure hypnozoite infections (White et al., 2018).

The factors underlying the development of $P$. vivax sporozoites into replicating schizonts or their persistence as hypnozoites and subsequent reactivation remain poorly defined. Key questions regarding the regulation of hypnozoite biology have focused on how it differs regionally, seasonally, and between strains. Relapse frequency varies by climate and geographical region, with temperate strains exhibiting long periods of latency and tropical ones relapsing at shorter intervals (Battle et al., 2014; Huldén et al., 2008; White, 2011). The observation that $P$. vivax may be able to regulate hypnozoite formation in accordance with environmental conditions feeds into a hypothesis that the developmental outcome within the liver may be pre-determined in the sporozoite (Lysenko et al., 1977; Ungureanu et al., 1976). In addition, observations in humanized rodent livers have identified sympatric $P$. vivax strains with stable differences in hypnozoite formation rates (Mikolajczak et al., 2015). This points to genetic heterogeneity among $P$. vivax sporozoites that may play a role in defining developmental fate, consistent with the tachy- and bradysporozoites proposed by Lysenko et al. (Lysenko et al., 1977). System-wide studies offer an opportunity to find evidence for sporozoite pre- 
programming; however, previous analyses of $P$. vivax sporozoites have been performed using bulk-sequencing approaches (Muller et al., 2019; Roth et al., 2018a; Westenberger et al., 2010) which obscure variation that might exist between individual parasites.

Single-cell RNA sequencing methods (scRNA-seq) constitute a recent advancement applicable for assessing parasite-to-parasite differences. ScRNA-seq has differentiated multiple transcriptomic states among individual $P$. berghei and $P$. falciparum sporozoites (Bogale et al., 2021; Howick et al., 2019; Real et al., 2021; Ruberto et al., 2021). However, the extent to which $P$. vivax sporozoites vary at the single-cell level has not been studied. Therefore, the application of scRNA-seq technology provides an opportunity to explore heterogeneity amongst $P$. vivax sporozoites and examine the existence of distinct transcriptional signatures that may help better understand the sporozoite's developmental fate.

In this study, we analyse the transcriptomes of 9,947 P. vivax sporozoites captured using droplet-based scRNA-seq technology. We first cross-reference the data with sporozoite bulk microarray and RNA-seq data to show consistent transcription of known genes upregulated in sporozoites. Next, we represent the data in low dimensional space and identify sporozoites in various transcriptomic states using both clustering and pseudotime trajectory methods. Finally, we perform comparative analyses with publicly available $P$. falciparum sporozoite and $P$. vivax blood-stage scRNA-seq data (Real et al., 2021; Sà et al., 2020) and highlight both conserved and unique gene usage patterns between sporozoites and erythrocytic forms. Overall, our work provides an important, new resource for the malaria community by offering key insights into gene usage among $P$. vivax sporozoites and the factors driving their developmental trajectory at a resolution unattainable with bulk transcriptomics.

\section{Results}

\section{Processing, alignment, and pseudo-bulk assessment of $\boldsymbol{P}$. vivax sporozoite scRNA-seq data}

Given its high-throughput capability and prior use for sporozoites from murine-infecting Plasmodium species (Bogale et al., 2021; Ruberto et al., 2021), we used the 10X Genomics gene expression platform to profile the transcriptomes of individual $P$. vivax sporozoites. We dissected and purified sporozoites from the salivary glands of An. dirus mosquitoes in triplicate, with each replicate comprising sporozoites derived from mosquitoes fed on a blood-meal from a different patient isolate. Sporozoites dissected and released from mosquito salivary glands were kept in Hank's Balanced Salt Solution at $4^{\circ} \mathrm{C}$ to minimize their activation. Sporozoites from each 
112 replicate were infectious to primary human hepatocytes, as indicated by their ability to generate

113 liver forms in a 384-well microtiter plate platform (Roth et al., 2018b)(Figures S1A-C). High-

114 content analysis of liver-stage cultures demonstrated that the sporozoites developed into

115 hypnozoites and schizonts at a ratio of $\sim 4: 5$ to $\sim 6: 4$, depending on the case (Figure S1B).

116 Between 5000-8000 sporozoites were loaded on a 10x Genomics' Chromium controller to

117 partition the sporozoites, lyse, and uniquely-tag transcripts. After tagging the transcripts,

118 Illumina compatible short-read libraries were generated and sequenced (Figure $1 \mathrm{~A}$ and Figure

119 S1D; Table S1).

After aligning the sequencing data to the $P$. vivax P01 genome (Figure S2A), various metrics confirmed that the libraries were of high quality (Figure S2B). An average of $48 \%$ of reads across all replicates $(638,820,734 / 1,340,791,021)$ mapped to the $P$. vivax P01 genome

123 (Figure 1B), with the remainder mapping to An. dirus (32\%; 437,925,660/1,340,791,021) or not mapping at all $(20 \% ; 264,044,627 / 1,340,791,021)$ (Figure S2C and Figure S2D; Table S2). transcriptome was a gap in knowledge that restricted accurate quantification of transcription.

127 Given the 10x Genomics' scRNA-seq technology captures specifically the 3' end of transcripts

128 (Klein et al., 2015; Macosko et al., 2015; Zheng et al., 2017), RNA with long untranslated regions (UTRs) may be sequenced and mapped to the genome but remain unquantified due to the incomplete annotation of these gene-flanking regions. This is a documented challenge for $3^{\prime}$ capture methods in Plasmodium and other non-model organisms (Bogale et al., 2021; Packer et al., 2019; Ruberto et al., 2021; Shields et al., 2021). However, a more complete $P$. vivax transcriptome, including UTRs, was recently reported (Siegel et al., 2020) and was incorporated into our alignment pipeline (Figure S2A). The inclusion of UTR coordinates resulted in a 1.3-fold increase of reads assigned to $P$. vivax genes (Figure 1B; Table S3). Compared to the alignment of reads using gene models lacking UTRs, UTR inclusion resulted in the detection of an additional 417 genes and an increase in counts for 1,182 genes (Figure S3A; Tables S2 and S3). The largest increase was found for the gene encoding gamete egress and sporozoite traversal protein (PVP01_125800) (Figure 1C; Table S3). Other notable examples of genes with increased transcription included serine threonine protein phosphatase $2 B$ catalytic subunit $A$

141 (PVP01_0117400), RNA-binding protein Musashi (PVP01_0715100) and a conserved protein of unknown function (PVP01_1011800) (Figure 1C; Table S3). 
bioRxiv preprint doi: https://doi.org/10.1101/2021.11.24.469176; this version posted November 24, 2021. The copyright holder for this preprint (which was not certified by peer review) is the author/funder, who has granted bioRxiv a license to display the preprint in perpetuity. It is made available under aCC-BY-NC-ND 4.0 International license.

A

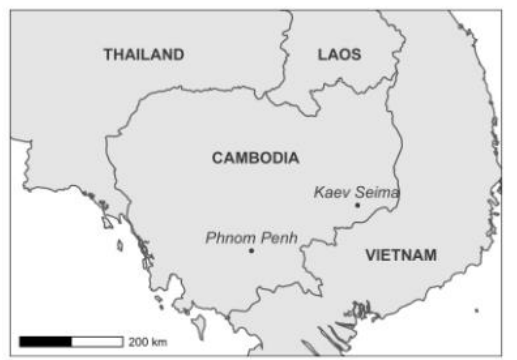

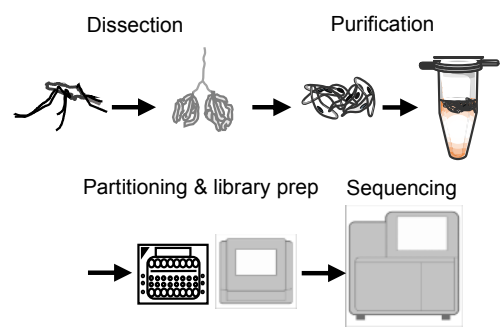

B

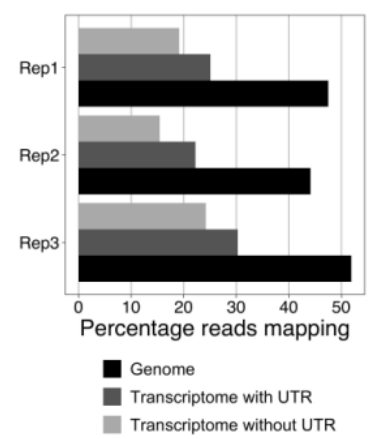

C
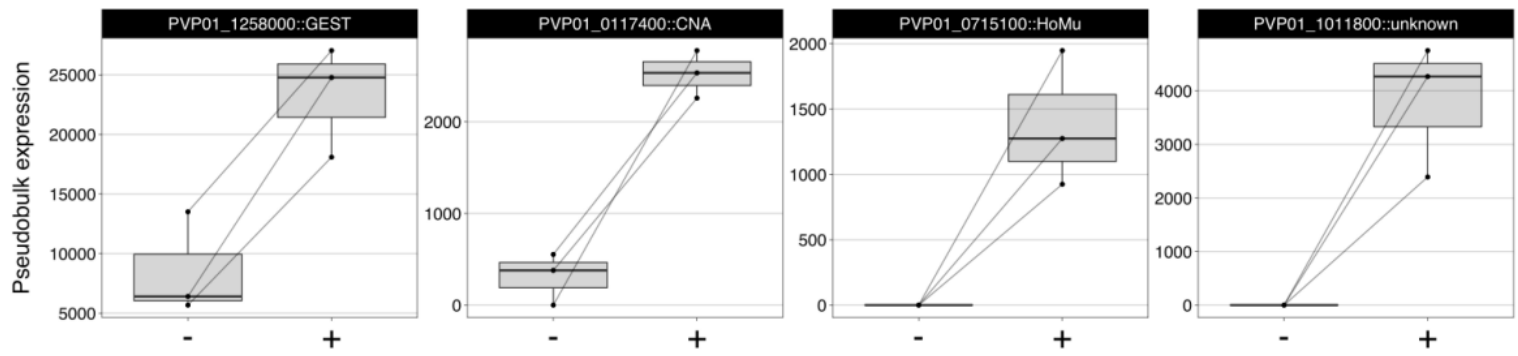

D

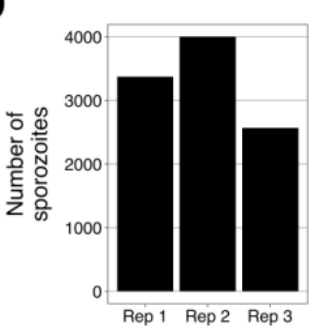

$\mathbf{F}$

E
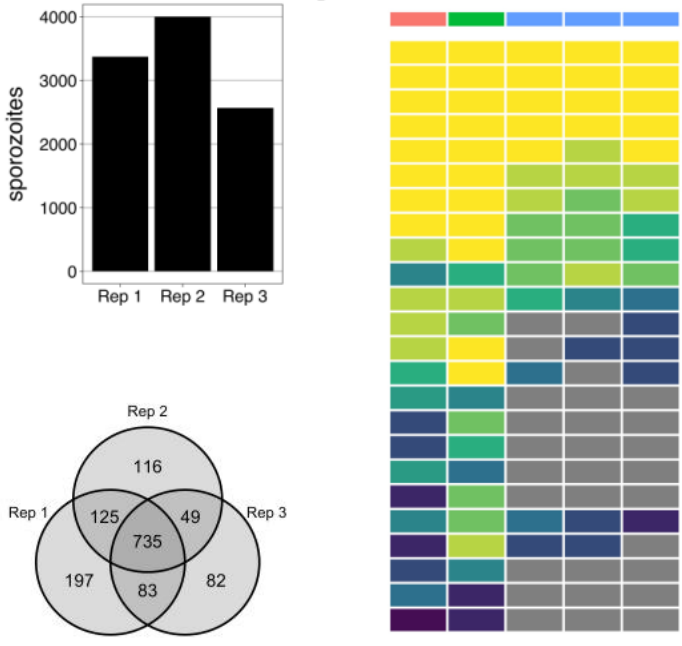

PVP01_0602100::early transcribed membrane protein PVP01_0934800::heat shock protein 70, putative

PVP01_1120200: : phosphatidylcholine-sterol acyltransferase, putative

PVP01_1242200::serine/threonine protein phosphatase UIS2, putative

PVP01_0305100::Iysine decarboxylase, putative

PVP01_1439100:: conserved Plasmodium protein, unknown function

PVP01_1403100::protein UIS3, putative

PVP01_0612400::merozoite capping protein 1, putative

PVP01_0212900::cysteine desulfurase, putative

PVP01_1325800::cyclophilin, putative

$\begin{array}{ll}\text { PVP01_1325800::cyclophilin, putative } & \text { Percentle } \\ \text { PVP01_0207300::eukaryotic translation initiation factor 2-alpha kinase 2, putative } & 0-10 \\ \text { PVP1-20 } & 11-20\end{array}$

PVP01_1223200.CPW-WPC k

PVP1_1259800::conserved protein, unknown function

PVP01_1458600::conserved Plasmodium protein, unknown function

PVP01_1015200::mRNA-binding protein PUF1, putative

PVP01_1249200::Iarge ribosomal subunit nuclear export factor, putative

PVP01_1019800::ATP-dependent DNA helicase UvrD, putative

PVP01_1302300::origin recognition complex subunit 1, putative

PVP01_1322200::lipase, putative

PVP01_0807800: zinc finger protein, putative

PVP01_1415800::TFIIH basal transcription factor subunit, putative

PVP01_0607200::RNA-binding protein, putative

PVP01_1250500:: conserved Plasmodium protein, unknown function

PVP01_1016400::F-actin-capping protein subunit beta, putative

Figure 1. Strategy used to assess $P$. vivax sporozoites transcriptomes at single-cell resolution.

(A) Schematic illustrating the geographical region, sample preparation and sequencing pipeline used to capture single-cell transcriptomes of $P$. vivax sporozoites. (B) Percentage of reads aligning to the $P$. vivax $\mathrm{P} 01$ genome and transcriptome (with- or without- UTR information) across the three replicates. (C) 'Pseudobulk' visualisation of UMI counts aligned to $P$. vivax P01 transcriptome without (-) or with (+) UTR information. (D) Number of sporozoite transcriptomes retained post cell- and gene- filtering. (E) Number of unique and overlapping genes detected across scRNA-seq datasets.(F) Comparison of orthologous up-regulted in infective sporozoites (UIS) genes (obtained from P.berghei) across three high-throughput sequencing technologies (microarray: Westenberger et al. 2009, bulk-RNA seq: Muller et al. 2019 and single-cell RNA seq; current study). To account for the differences in the total number of genes detected across technologies, gene expression values were compared using percentile ranks. GEST: gamete egress and sporozoite traversal; CNA: serine/threonin protein phosphatase 2B catalytic subunit A; HoMu: RNA-binding protein Musashi; UTR: untranslated region.

We next assessed the transcriptomes of 9,947 sporozoites obtained across all replicates

144 (Figure 1D). Of the 1,387 genes detected, 735 (53\%) were detected in all three replicates

145 (Figure 1E). We observed high correlation in the transcription of genes detected in each 
146 replicate (mean Pearson correlation coefficient, $R=0.94, p<0.05$; Figure S3B). Genes

147 encoding for circumsporozoite protein (PVP01_0835600), gamete egress and sporozoite

148 traversal protein (gest; PVP01_1258000), and sporozoite protein essential for cell traversal

149 (spect; PVP01_1212300) were among those with the highest transcription (Table S4).

150 Comparative analyses of our data with bulk transcriptomic studies (Muller et al., 2019;

151 Westenberger et al., 2010) revealed consistent detection of various genes implicated in

152 sporozoite biology (Figure 1F). These results paint a clearer picture of gene usage in $P$. vivax

153 sporozoites by incorporating each genes' UTRs and highlight the capacity of scRNA-seq in

154 assessing transcription in $P$. vivax sporozoites.

Assessment of $\boldsymbol{P}$. vivax sporozoites at single-cell resolution reveals transcriptomic heterogeneity

Our assessment of various per-cell metrics revealed differences in $P$. vivax sporozoites at the transcript level, specifically in the distribution of unique molecular identifiers (UMIs); the absolute number of transcripts (Kivioja et al., 2011), and genes detected in individual sporozoites across replicates (Figure 2A). Using Uniform Manifold Approximation Projection

161 (UMAP) of the data to assess the transcriptomic differences visually, we found three distinct 162 populations of sporozoites (Figure 2B). Overlaying the UMI and genes detected data on the 163 UMAP revealed sporozoites in two main transcriptomic states: forms with higher gene usage 164 (represented by the cells on the left side of the UMAP) and forms with lower gene usage (on the 165 right) (Figure 2C). We first visualized transcription of well-described sporozoite membrane166 associated and cell traversal proteins (Figure 2D) (Coppi et al., 2011; Ishino et al., 2004;

167 Matuschewski et al., 2002; Mueller et al., 2005; Real et al., 2017; Sinnis et al., 1994; Talman et 168 al., 2011). Next, given that $P$. vivax parasites can form both replicating schizonts and

169 hypnozoites in the liver, we assessed the transcription profiles of genes implicated in sporozoite 170 developmental fating (Muller et al., 2019). However, no clear pattern was observed, and 171 sporozoites in both populations transcribed genes encoding for membrane proteins, cell 172 traversal and initiation of invasion, and translational repression (Figure 2D). 
bioRxiv preprint doi: https://doi.org/10.1101/2021.11.24.469176; this version posted November 24,2021 . The copyright holder for this preprint (which was not certified by peer review) is the author/funder, who has granted bioRxiv a license to display the preprint in perpetuity. It is made available under aCC-BY-NC-ND 4.0 International license.

A
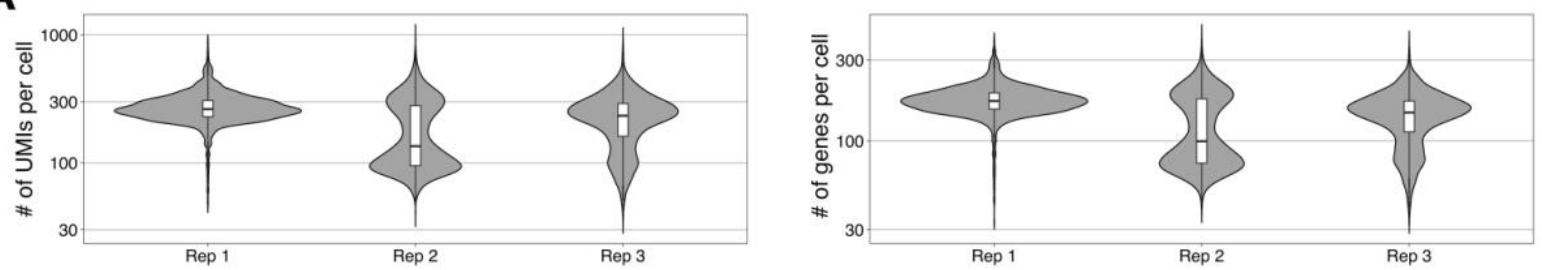

B

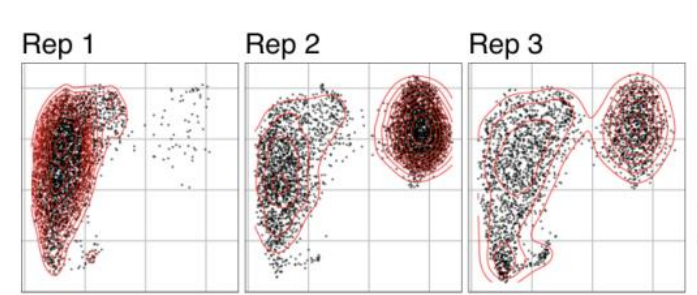

C

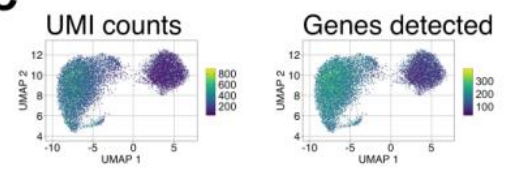

D
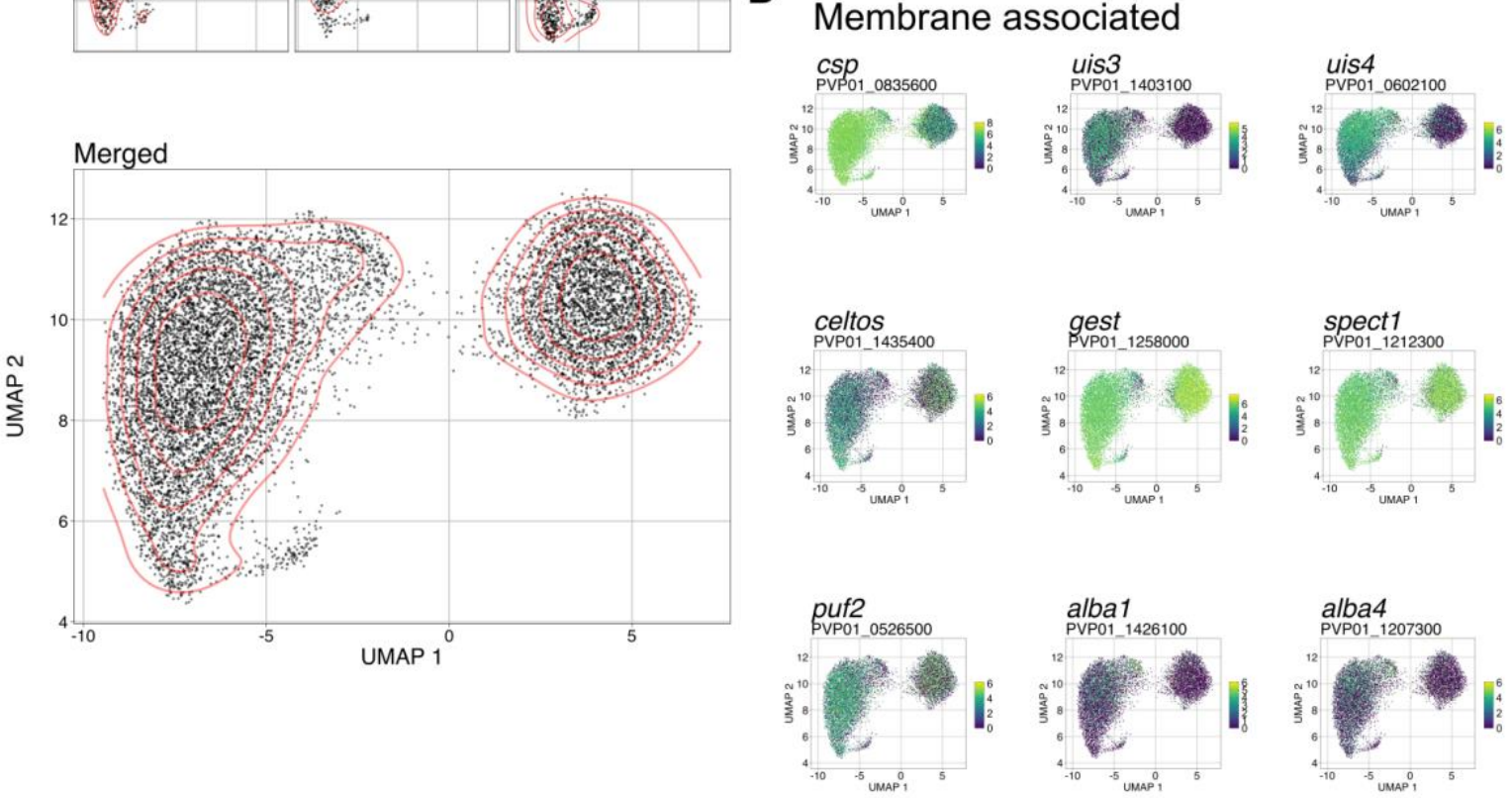

Figure 2. Analysis of $P$. vivax sporozoite gene expression at single-cell resolution.

(A) Violin plots (with boxplox overlaid) showing the distribution of unique molecular identifiers (UMIs) per cell (left), and genes detected be cell (right). (B) UMAPs of $P$. vivax sporozoite transcriptomes, visualized per replicate (upper) and combined (lower). Red-lines represent the density of cells represented in low dimensional space. (C) UMAPs of $P$. vivax transcriptomes colored by total number of UMIs (left) and total number of genes detected (right) per cell. (D) UMAPs of $P$. vivax sporozoite transcriptomes. Cells colored by expression of various genes implicated in sporozoite biology. Scale bar: normalised expression. csp: circumsporozoite protein; uis3: upregulated-in-infective sporozoites 3; uis4: upregulated-in-infective sporozoites 4; celtos: cell-traversal protein for ookinetes and sporozoites; gest: gamete egress and sporozoite traversal; spect1: sporozoite microneme protein essential for cell traversal 1; puf2: mRNA-binding protein PUF2; alba1: DNA/RNA binding protein Alba1; alba4: DNA/RNA binding protein alba4. unsupervised graph-based clustering algorithm (Traag et al., 2019) with a conservative grouping parameter (Figure S4A) to systematically divide the sporozoites into three transcriptional

177 clusters (Figure 3A), consisting of 86 (cluster C1), 6,982 (cluster C2) and 2,879 (cluster C3) sporozoites, respectively. The number of sporozoites in each cluster varied across replicates, indicating their variability across different mosquito infections (Figure 3B). We next identified 
bioRxiv preprint doi: https://doi.org/10.1101/2021.11.24.469176; this version posted November 24, 2021. The copyright holder for this preprint (which was not certified by peer review) is the author/funder, who has granted bioRxiv a license to display the preprint in perpetuity. It is made available under aCC-BY-NC-ND 4.0 International license.

markers that define the sporozoites in each cluster using the FindAllMarkers function in Seurat (Stuart et al., 2019). We defined a marker as a gene detected in over $30 \%$ of cells in a given

182 cluster and displaying significantly greater transcription than the other clusters. In total, we

183 found 159 markers (adjusted p-value < 0.05, Table S5). Notably, sporozoite-specific protein,

184 S10 (PVP01_0304200), was a marker for sporozoites in C1 (Figure 3C; Table S5), and has

185 previously been shown to be highly transcribed in midgut sporozoites (Bogale et al., 2021;

186 Ruberto et al., 2021). C2 markers included circumsporozoite protein (PVP01_0835600); early

187 transcribed membrane protein (PVP01_0602100); TRAP-like protein (PVP01_1132600); and

A

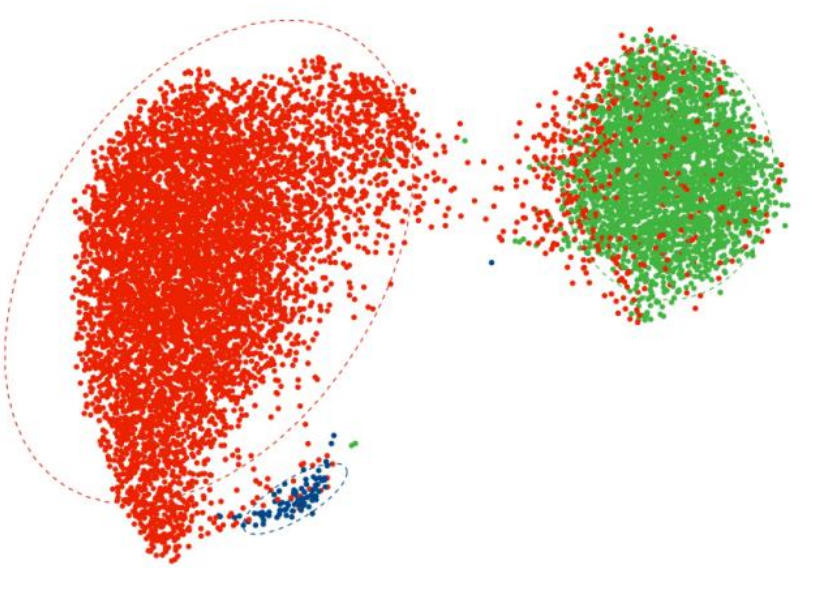

C

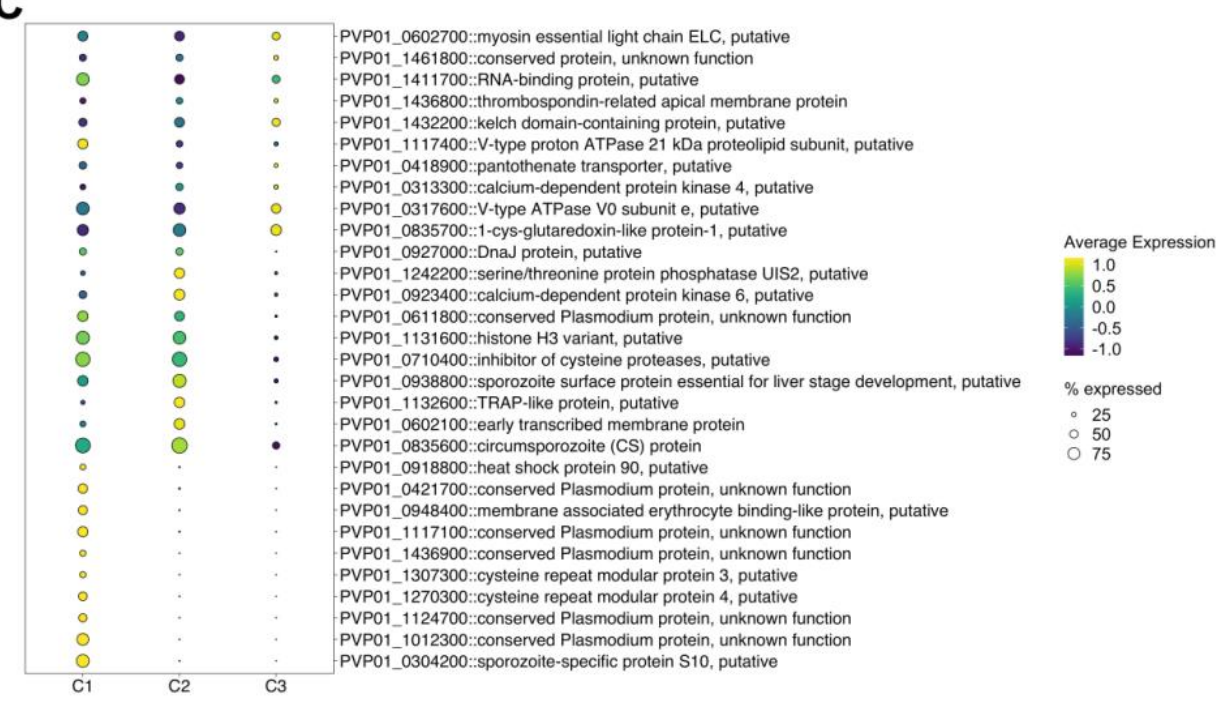

B
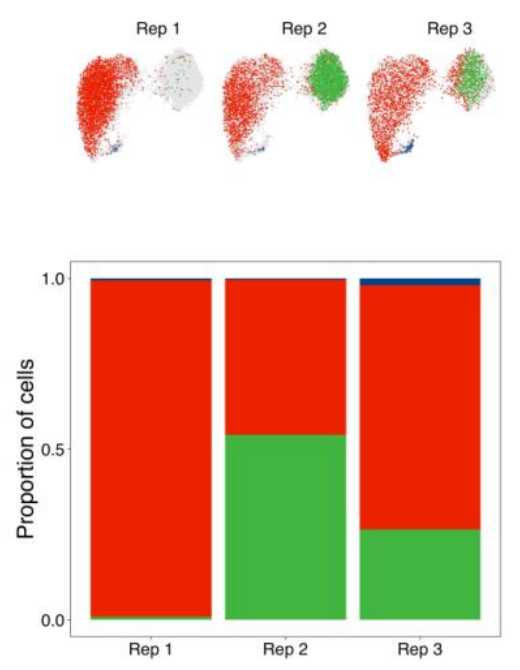

Figure 3. Clustering and differential gene expression analysis of $P$. vivax sporozoites.

(A) UMAP of integrated $P$. vivax sporozoite transcriptomes coloured by cluster (Leiden algorithm; resolution parameter $=0.1$ ). (B) UMAP of integrated $P$. vivax sporozoite transcriptomes split by replicate (upper) and the percentage of sporozoites in each cluster from the replicate (lower). (C) Dot plot showing topmarkers that distinguish each of the three clusters. The size of the dot corresponds to the percentage of sporozoites expressing the gene. Scale bar: normalised expression, scaled. 
sporozoite surface protein essential for liver stage development (PVP01_0938800) (Figure 3C; Table S5). Relative to C1 and C2, C3 had the fewest markers (20) (adjusted p-value < 0.05), and of the markers identified, their changes in transcription were modest (average $\log _{2}$ - fold- $^{-}$ change range $0.28-0.84$; Figure S4B). Markers in this cluster included genes involved in proton transport (PVP01_0317600 and PVP01_1117400); redox response (PVP01_0835700 and PVP01_1249700); and heat shock proteins (PVP01_1440500 and PVP01_1011500) (Figure 3C; Table S5).

\section{Trajectory-based pseudotime analysis reveals various transcription patterns in} sporozoites

A caveat of using a cluster-based classification method is that cells are forced into groups. In developing systems, such as sporozoites, cell transitions may be occurring more continuously. The modest number of markers defining each cluster (Table S5) suggested that a continuum of transcriptional states may exist. We thus examined the transcriptional profiles of $P$. vivax sporozoites in the context of pseudotime. We used Slingshot (Street et al., 2018) to construct a trajectory through the cells (Figure 4A). We observed differences in the distribution of sporozoites among replicates over pseudotime. Sporozoites from replicate 1 were unimodal and primarily enriched earlier in the trajectory, whereas sporozoites from replicates 2 and 3 were bimodal, with more cells near the end of the trajectory (Figure 4B).

Next, to assess changes in transcription as cells progressed along the trajectory, we modelled the transcription of each gene as a function of pseudotime (van den Berge et al., 2020). We identified 1072 differentially transcribed genes (False discovery rate (FDR) $<0.01$;

\section{Table S6). Heatmap visualization of the data revealed three common patterns of how} transcription changed over pseudotime, including a gradual decrease, transient increase, and transient decrease (Figure 4C). Our assessment of $P$. vivax sporozoites over pseudotime provides further insights into gene usage, taking into account that cell transitions in these forms may occur more continuously. 
bioRxiv preprint doi: https://doi.org/10.1101/2021.11.24.469176; this version posted November 24, 2021. The copyright holder for this preprint (which was not certified by peer review) is the author/funder, who has granted bioRxiv a license to display the preprint in perpetuity. It is made available under aCC-BY-NC-ND 4.0 International license.

A

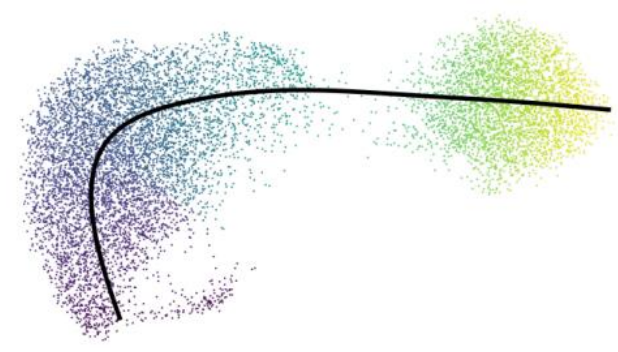

C
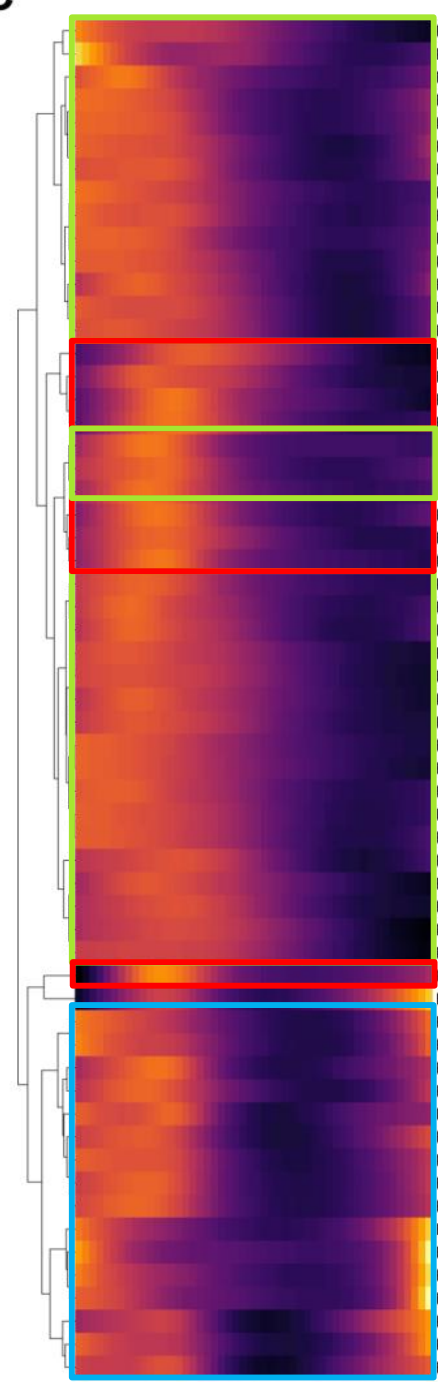

B

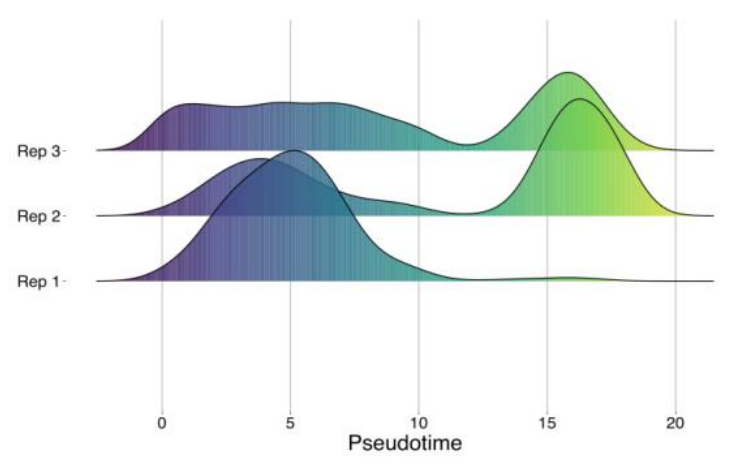

High

VP01_1213900::sodium-dependent phosphate transporter, putative PVP01_1463200: actin, putative

PVP01_0319000:: conserved protein, unknown function

PVP01_0505600::GPI-anchored micronemal antigen

PVP01_0917500: : conserved protein, unknown function

PVP01_1014200: FoP domain-containing protein, putative

PVP01_1422800: conserved Plasmodium protein, unknown function

PVP01 1242100: vacuolar ATP synthase subunit d, putative

PVP01_1344100::cysteine-rich small secreted protein CSS, putative PVP01_1111200: falcilysin, putative

PVP01 1315100:: conserved protein, unknown function

PVP01_0516700:: BEM46-like protein, putative

PVP01_0515400: : heat shock protein 70, putative

PVP01_0515400:: heat shock protein 70, Put

PVP01_0517100: $: 14-3-3$ protein, putative
PVP01_0602100::early transcribed membrane protein

PVP1_ 1242200::serine/threonine protein phosphatase UIS2, putative

VVP01_0532200: :conserved Plasmodium protein, unknown function

VP01_1343900: :conserved Plasmodium protein, unknown function

PVP01_0526500::mRNA-binding protein PUF2, putative

PVP01_0712500: conserved Plasmodium protein, unknown function

PVP1 1405700: NLL interacting factor-like phosphatase, putative

PVP01_0807700: :histone deacetylase 2, putative

PVP01_0934800: :heat shock protein 70, putative

PVP01_1132600::TRAP-like protein, putative

PVP01_0303900: 6-cysteine protein, putative, pseudogene

PVP01 0614100: conserved Plasmodium protein, unknown function

PVP01_1308600::cGMP-specific 3', 5'-cyclic phosphodiesterase alpha, putative

PV01_0611800: : conserved Plasmodium protein, unknown function

VVP1_1131600: histone $H 3$ variant, putative

PVP1_1144400::GYF domain-containing protein, putative

PVP01_1427300: protein KIC7, putative

VP01_0117400::serine/threonine protein phosphatase 2B catalytic subunit A, putative

PVP01_0934200: apical membrane antigen 1

PVP01_0938800::sporozoite surface protein essential for liver stage development, putative

PVP01_1022600::conserved Plasmodium protein, unknown function

PVP01_1225100::AMP deaminase, putative

PVP01 1460700: translation initiation factor SUI1, putative

PVP01_0923400 calcium-dependent protein kinase 6, putative

PVP01_1210400" conserved Plasmodium protein, unknown function

PVP01 0710400 : : inhibitor of cysteine proteases, putative

PVP01_0835600: : circumsporozoite (CS) protein

PVP1_0912600:: peptidyl-prolyl cis-trans isomerase, putative

PVP1_ 1453200:: conserved protein, unknown function

PVP01_0317600::V-type ATPase V0 subunit e, putative

PVP1_0317600::V-type ATPase V0 subunit e, putative

VP 01_1120200: phosphatidylcholine-sterol acyltransferase, putative

VP01_1461700: ADP-ribosylation factor GTPase-activating protein 1, putative

PVP01_0835700:1-cys-glutaredoxin-like protein-1, putative

PVP01_1456100: COPI associated protein, putative

VP01_1212300::sporozoite protein essential for cell traversal, putative

VPP01_1110200::glideosome-associated connector, putative

PVP01 1249700: thioredoxin 1, putative

PVP01 $0611700:$ :conserved Plasmodium protein, unknown function

PVP1_0210700: thrombospondin-related sporozoite protein, putative

PVP01_0730900: proflin, putative

PVP1_1432200: :kelch domain-containing protein, putative

VVP1 1413500:: conserved Plasmodium protein, unknown function

VPP1 0412300 : secreted protein with altered thrombospondin repeat domain, putative

PVP01_1258000::gamete egress and sporozoite traversal protein, putative

Figure 4. Pseudotime analysis of $P$. vivax sporozoites.

(A) UMAP P. vivax sporozoite transcriptomes coloured by progression along pseudotime. Colour scale matches pseudotime in panel B; where darker cells are representative of cells placed earlier in pseudotime and lighter cells are projected later along the pseudotime trajectory. (B) Distribution of cells along pseudotime faceted by replicate. (C) Heatmap showing genes differentially express across pseudotime (Data shown: mean pseudobulk $>2000$, waldStat $>200$ and FDR $<0.01$ ). Genes are hierarchically clustered based on their pseudotime expression profile. Scale bar: normalized expression. 


\section{$P$. vivax sporozoites have distinct transcriptomic signatures relative to $P$. falciparum sporozoites}

Whereas $P$. vivax sporozoites can form hypnozoites in hepatocytes, $P$. falciparum sporozoites cannot. Therefore, we compared sporozoite transcriptomes from these two species to assess the extent to which their gene expression profiles differ and search for signatures of potential importance for hypnozoite formation. We first identified orthologous gene groupings to allow for the cross-species comparison (Emms and Kelly, 2019). After subsampling the $P$. vivax data to balance the proportions of $P$. falciparum (Real et al., 2021) and $P$. vivax cells, we then combined the two species' datasets to produce an integrated UMAP (Figure 5A top). The projection revealed that sporozoites in the $P$. vivax and $P$. falciparum data mapped close together (Figure 5A top). The projection also revealed distinct populations of $P$. vivax and $P$. falciparum cells, with the latter grouped by anatomical location in the mosquito (Figure $5 \mathrm{~A}$ bottom). The annotated $P$. falciparum data by mosquito location and activation status allowed us to infer the transcriptomic status of $P$. vivax sporozoites. Using these annotations as a guide, we grouped the sporozoites into four clusters (Figure 5B). Cluster 1 (ORTHO_C1) represented midgut/recently invaded sporozoites; cluster 2 (ORTHO_C2), salivary gland sporozoites; and clusters 3 and 4 (ORTHO_3 and ORTHO_4), activating or activated sporozoites. More than half of the $P$. vivax sporozoite data $(63 \%, 949 / 1500)$ clustered with the $P$. falciparum salivary gland sporozoites in ORTHO_C2 (Figure 5C and Figure S5A). Interestingly, some $P$. vivax sporozoites were assigned to clusters ORTHO_C1, ORTHO_C3 and ORTHO_C4, shedding light on their intrinsic heterogeneity within the salivary glands of the mosquito (Figure $5 \mathrm{C}$ and Figure S5A).

For each cluster, we identified genes that display greater transcription relative to the other clusters and are conserved in both species, which we denote as conserved markers. In total, we identified 12 conserved markers (adjusted p-value < 0.05; Figure S5C; Table S8).

Relative to the other clusters, only sporozoites in the midgut/recently invaded and salivary gland clusters (ORTHO_C1 and ORTHO_C2, respectively) had conserved gene signatures. We reasoned that the disproportionate number of sporozoites for each species in ORTHO_C3 and ORTHO_C4 (Figure 5C and Figure S5A) contributed to the lack of conserved markers detected. Of the genes with known function identified in ORTHO_C1, notable examples included sporozoite invasion-associated protein 1 (PVP01_0307900 | PF3D7_0408600), important for sporozoite exit from the mosquito midgut and colonization of the salivary gland (Engelmann et al., 2009) and sporozoite-specific protein S10 (PVP01_0304200 | PF3D7_0404800), a marker 
248

249

250

251

252

253

254

255

256

257

258

259

260

261

262

263

264

265

266

267

268

269

270

271

272

273

274

275

276

277

278

279

280

implicated in salivary gland invasion (Bogale et al., 2021; Ruberto et al., 2021) (Figure S5C;

Table S6). In ORTHO_C2, genes linked to invasion (PVP01_1132600 |

PF3D7_0616500::TRAP-like protein) and liver stage development (PVP01_0938800 |

PF3D7_1137800::SPELD) were among the conserved markers identified (Figure S5C; Table S8).

To elucidate species-specific transcription patterns within each cluster, we compared transcript levels between species. Each cluster contained orthologs transcribed solely in $P$. vivax or P. falciparum (Figure 5D left and Figure S5B; Table S9). ORTHO_C4, which was composed almost entirely of $P$. vivax sporozoites, was excluded from the analysis. We thus focused our analyses on the genes detected in both species in clusters one through three (Figure 5D right and Figure S5B; Table S9). In total, we identified 155 differentially transcribed genes (adjusted p-value < 0.05$), 44(28 \%)$ of which had unknown function (Table S10). In Figure S6A, we highlight the top differentially transcribed genes within each cluster. At a broad level, genes associated with ion transport (PVP01_0317600 | PF3D7_0721900, PVP01_1014700 | PF3D7_0519200, PVP01_1117400 | PF3D7_1354400, PVP01_1242100 | PF3D7_1464700) displayed greater transcription in ORTHO_C1 P. vivax sporozoites (adjusted p-value < 0.05, Table S10). Alternatively, clusters ORTHO_C2 and ORTHO_C3, various genes associated with locomotion and motility (PVP01_1132600 |PF3D7_0616500, PVP01_1435400 | PF3D7_1216600, PVP01_1218700|PF3D7_1335900) displayed greater transcription in $P$. falciparum (adjusted p-value < 0.05, Table S10). Combined with the species-specific genes identified in each cluster, these differences highlight the distinct transcriptomic signatures between $P$. vivax and $P$. falciparum.

ORTHO_C3 and ORTHO_C4 contained sporozoites with transcriptomic signatures similar to $P$. falciparum activated sporozoites. As ORTHO_C4 was made up of primarily $P$. vivax cells, we sought to assess the extent to which sporozoites in this cluster differed from those in ORTHO_C3 containing both $P$. falciparum and $P$. vivax sporozoites. In total, we identified 98 differentially transcribed genes (adjusted p-value $<0.05$, Table S11), several $(38 / 98 ; 38 \%)$ of which have no known function. Interestingly, genes that displayed significantly greater transcription in ORTHO_C4 (Figure 5E) contained Pfam domains associated with translational machinery (PF01200 and PF01253) and RNA-binding proteins (PF00076, PF00806), including mRNA-binding protein PUF2 (PVP01_0526500). PUF2 is an important eukaryotic cell-fate regulator (Zhang et al., 1997), and plays a key-role in translationally repressing transcripts, including uis3 and uis4, required during liver-stage development (Gomes-Santos et al., 2011; 
bioRxiv preprint doi: https://doi.org/10.1101/2021.11.24.469176; this version posted November 24,2021 . The copyright holder for this preprint (which was not certified by peer review) is the author/funder, who has granted bioRxiv a license to display the preprint in perpetuity. It is made available under aCC-BY-NC-ND 4.0 International license.

A
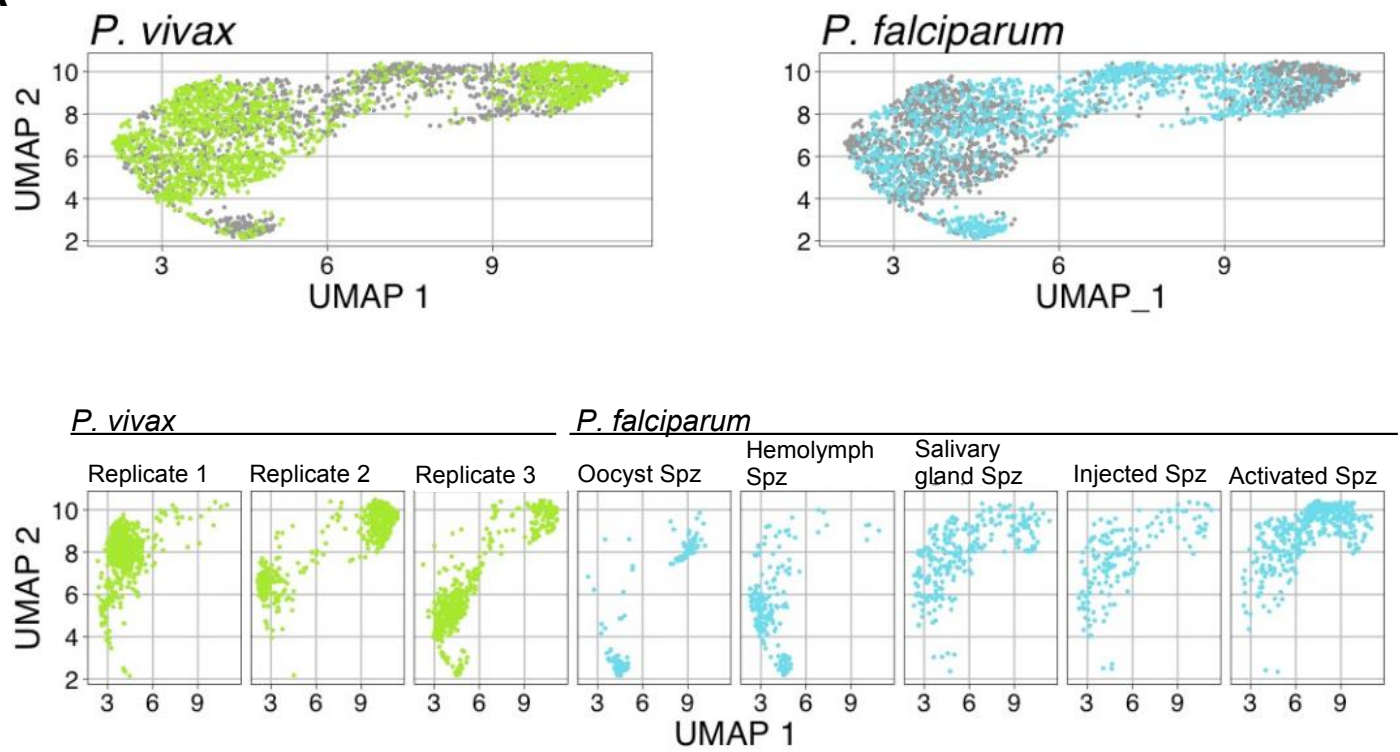

B

C
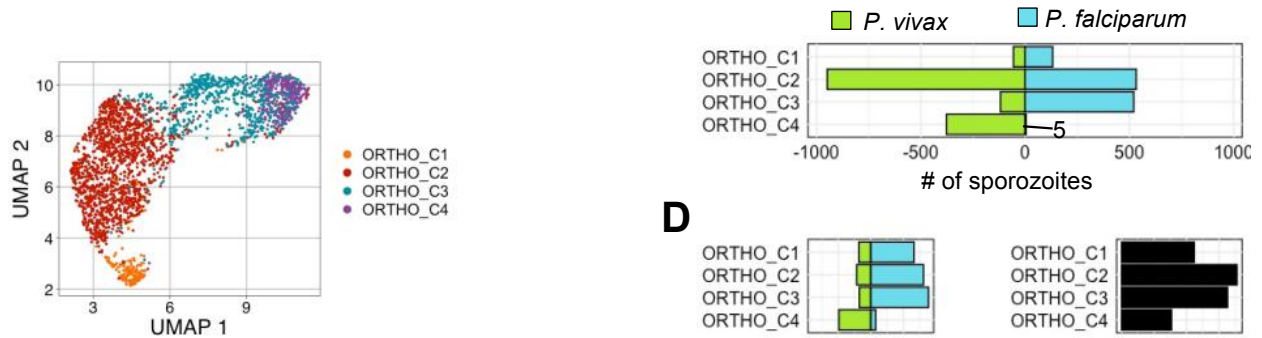

D

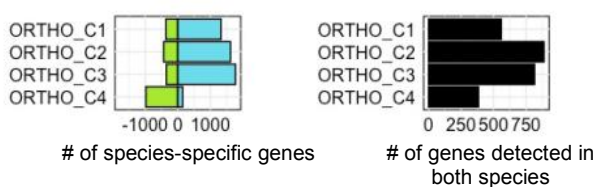

E

pFam Domains

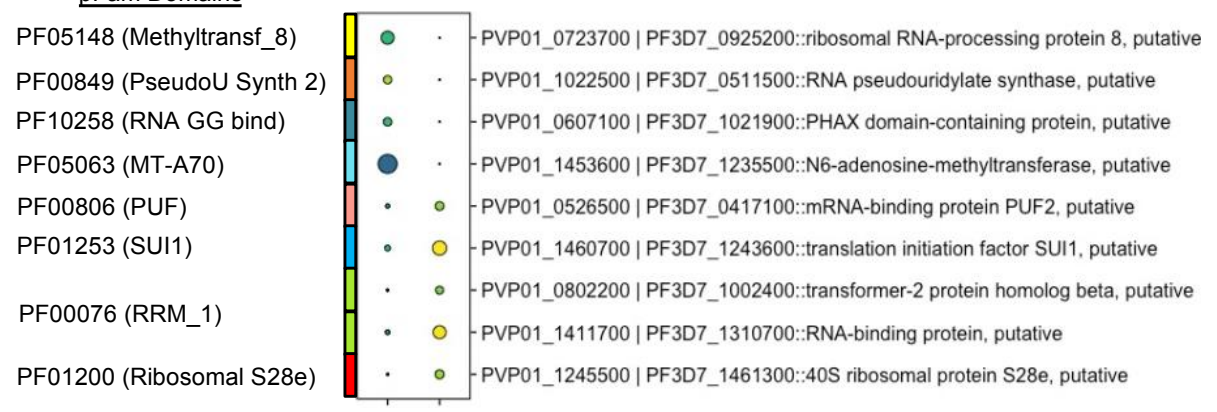

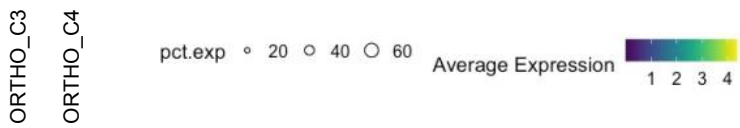

Figure 5. Integration of $P$. vivax and $P$. falciparum sporozoite datasets.

(A) UMAPs of integrated $P$. vivax and $P$. falciparum data. Top: split and coloured by species. Bottom: split by replicate $(P$.vivax) or sporozoite status ( $P$. falciparum). (B) UMAP of integrated $P$. vivax and $P$. falciparum data coloured by cluster. (C) Number of cells contributing to each cluster from $P$. vivax and $P$. falciparum samples. (D) Number of species-specific genes (left) and conserved one-to-one orthologs (right) detected in each cluster. (E) Differentially expressed genes between clusters ORTHO_C3 and ORTHO_C4 with known Pfam domains. . Scale bar: normalized expression.

281 Lindner et al., 2013). Interestingly, in the absence of PUF2 (Pfam: PF00806::PUF RNA binding repeat), salivary gland sporozoites may initiate exoerythrocytic development independently of the transmission-associated environmental cues (Gomes-Santos et al., 2011). These findings 
indicate although cellular morphology and invasion strategies are similar in sporozoites from the two species, their respective cellular states, as defined by their transcriptomic signatures, can vary drastically. Whether these differences in transcription play a role in determining the sporozoite's developmental fate upon reaching the liver warrants further investigation.

\section{Comparative analyses of $\boldsymbol{P}$. vivax sporozoite and blood-stage transcriptomes reveals} conserved and stage-specific signatures

To assess gene usage across different stages of the parasite's life cycle, we compared our sporozoite data with publicly-available scRNA-seq data from $P$. vivax blood stages (Sà et al., 2020). Before data integration, we realigned the blood-stage sequencing data to account for the UTR additions to the $P$. vivax gene models. As expected, we found a 1.4-fold gain in the number of reads mapping to gene loci when the UTRs were included in the alignment (Figure $S 7 A)$. Using the cell and gene filtering pipeline established for the sporozoite scRNA-seq data, 12,469 blood-stages parasites were assessed (Figure S7B). We detected a median of 682 genes and 1,442 UMIs per cell (Figure S7C). Furthermore, low-dimensional representation of the data confirmed distinct transcriptomic signatures across each sample (Figure S7D).

After cell and gene filtering, we integrated the $P$. vivax blood-stage data with our sporozoite data (Figure 6A, Figure S7E) and performed differential transcription analysis. We identified 208 differentially transcribed genes, 36 of which remain uncharacterized (minimum expression in $50 \%$ of cells, adjusted p-value $<0.1$; Table S13). Among the differentially transcribed genes, 59 displayed sporozoite-specific transcription (adjusted p-value $<0.01$; Table S13). As highlighted in Figure 6B, sporozoite-specific markers encoded for transcripts involved in sporozoite development, maturation, and host-cell infection (Al-Nihmi et al., 2017; Cerami et al., 1992; Coppi et al., 2011; Ishino et al., 2004; Sinnis et al., 1994; Talman et al., 2011).

Furthermore, we identified a small proportion of transcripts $(18 / 208,8 \%)$ present in a similar percentage of sporozoite and erythrocyte forms but with significantly higher transcriptional abundance in sporozoites (Figure 6C; Table S13). Together, this integrated analysis reveals various stage-specific markers and provides the framework for creating a comprehensive reference map for $P$. vivax. 
bioRxiv preprint doi: https://doi.org/10.1101/2021.11.24.469176; this version posted November 24, 2021. The copyright holder for this preprint (which was not certified by peer review) is the author/funder, who has granted bioRxiv a license to display the preprint in perpetuity. It is made available under aCC-BY-NC-ND 4.0 International license.

A

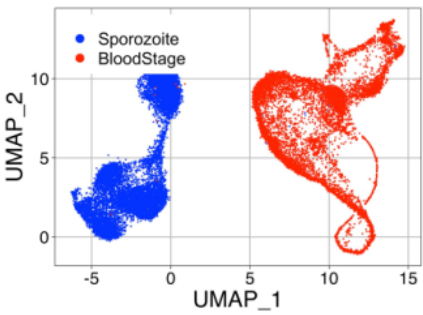

B

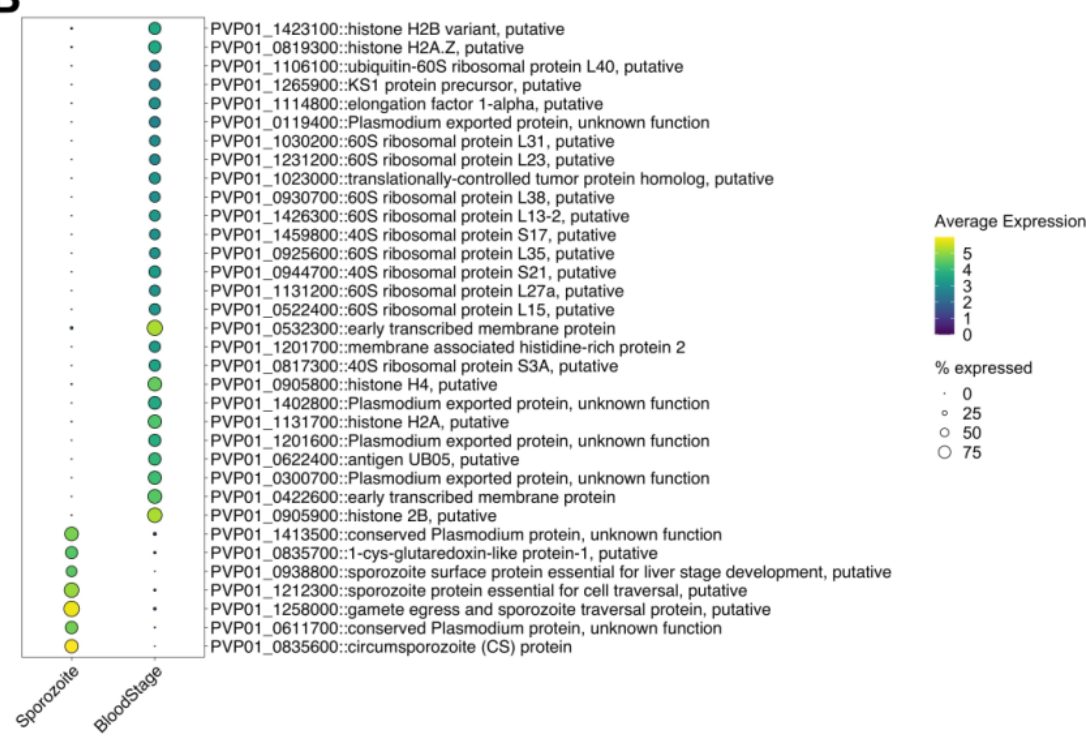

C

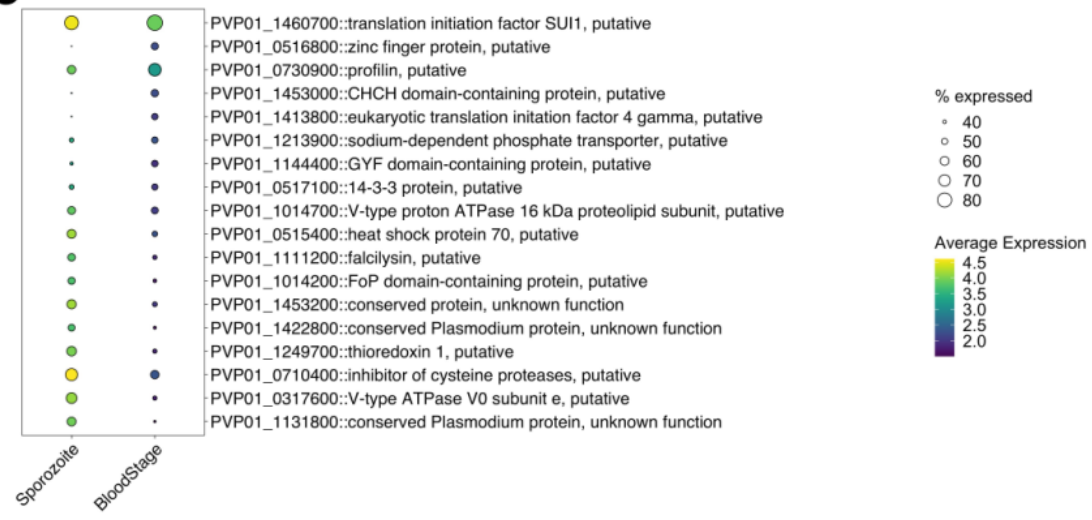

Figure 6. Integration and comparative analyses of $P$. vivax sporozoite and blood-stage parasite transcriptomes.

(A) UMAP of integrated $P$. vivax sporozoite and blood-stage parasite transcriptomic data. (B) Dot plot highlighting the top differentially expressed genes with stage-specific specific expression patterns (absolute difference in gene detection $>65 \%$ between sporozoites and blood stages). (C) Dot plot highlighting the top differentially expressed genes with detection in similar percentages of cells between sporozoites and blood-stage parasites. Scale bar: normalized expression. 


\section{Discussion}

314

315

316

317

318

319

320

321

322

323

324

Our scRNA-seq data reveals transcriptional differences among $P$. vivax sporozoites at a resolution previously unattainable with bulk transcriptome-wide approaches. As is expected with droplet-based single-cell capture technologies, a comparison of our scRNA-seq data with that of bulk methods reveals a reduced overall number of genes detected using the single-cell approach (Haque et al., 2017; Muller et al., 2019; Westenberger et al., 2010). However, we find that the detection of highly transcribed sporozoite genes is achieved across technologies. Herein we also describe improved approaches for processing and analyzing scRNA-seq data useful for the wider community. First, our overall gene detection rate was dramatically improved by including each genes' UTRs in our alignment strategy. Second, clustering and pseudotime analyses were useful for providing new insights into transcription patterns amongst $P$. vivax sporozoites in the salivary glands of mosquitos.

Visualizing the data in low-dimensional space reveals transcriptional heterogeneity amongst sporozoites. After assigning the sporozoites to clusters and performing differential transcriptional analysis, we identified annotated and unannotated genes underlying this heterogeneity. We found that some sporozoites isolated from the salivary glands show increased transcriptional activity of orthologous genes previously shown to be transcribed in $P$. berghei midgut sporozoites (Bogale et al., 2021; Ruberto et al., 2021). We interpret the detection of these markers in $P$. vivax to represent sporozoites that have recently invaded the salivary glands. However, we also found sporozoites that display lower gene usage, which we hypothesize is indicative of maturation. The pseudotime analysis serves to complement the cluster-based assessment of sporozoites. The ordering of sporozoites reveals a gradual decrease in the transcription of most genes. These findings likely recapitulate the transcription changes occurring as the sporozoite transitions from a recently invaded salivary gland sporozoite to a mature one. Translational repression of mRNA is one molecular mechanism sporozoites use to ensure timely production of proteins upon entering its host (reviewed in (Briquet et al., 2021; Schäfer et al., 2021)). Our observation of the low number of UMIs and genes detected per cell, which corresponds with other assessments of Plasmodium sporozoites at single-cell resolution (Bogale et al., 2021; Howick et al., 2019; Real et al., 2021; Ruberto et al., 2021), suggests that transcriptional repression may also play a role in the sporozoites' preparation for host invasion. 
It has been hypothesized that $P$. vivax sporozoites are pre-programmed to become

345

346

347

348

349

350

351

352

353

354

355

356

357

358

359

360

361

362

363

364

365

366

367

368

369

370

371

372

373

374

375

376

replicating schizonts or hypnozoites (Lysenko et al., 1977; Ungureanu et al., 1976). However, evidence to support this hypothesis at the molecular level is lacking. We proposed that the transcriptomic analysis of individual sporozoites could test this hypothesis and might support identification of two distinct sporozoite populations, with one destined for normal hepatic development and the other for hypnozoite formation. By comparison, we expected sporozoites from $P$. falciparum, which do not form hypnozoites, would have the first but lack the second population. In this regard, the results of our study are interesting, but, at this stage, not conclusive. Our comparative analyses identify a large population of $P$. vivax and $P$. falciparum sporozoites that have conserved patterns in gene usage for various markers implicated in sporozoite biology. These include the genes encoding for sporozoite invasion-associated protein 1, TRAP-like protein, and sporozoite surface protein essential for liver stage development (Al-Nihmi et al., 2017; Engelmann et al., 2009; Heiss et al., 2008). Interestingly, distinguishing $P$. vivax from $P$. falciparum were a subset $(\sim 25 \%)$ of $P$. vivax sporozoites transcribing genes (e.g. puf2) associated with translational regulation and repression. Noting the role translational repression through RNA-binding proteins, including PUF2, play developmental fating in eukaryotes (Kong and Lasko, 2012), it is intriguing to speculate on possible links between this population and the bradysporozoites (Lysenko et al., 1977) proposed to be destined for hypnozoite formation. This population is potentially of a sufficient size to account for the proportion of liver forms found to be hypnozoites in vitro (47-61\%). However, it is not possible to conclude any link between these cells and liver-stage development at this stage. Clearly, further experimentation will be required to resolve this issue.

Future analyses comparing $P$. vivax to the other sporozoites derived from parasites that cause relapsing malaria, such as $P$. cynomolgi and $P$. ovale, may help shed light on the relapsing-specific factors associated with the parasite's developmental fate. However, the differences in methodology used to generate sporozoite scRNA-seq datasets should be considered for these future studies. Of note, scRNA-seq of $P$. falciparum sporozoites was performed after dissecting salivary glands into Schneider's insect medium and then sequencing individual sporozoites following Accudenz purification, immunofluorescent staining and fluorescence-activated cell sorting (Real et al., 2021). In this study we used only a density gradient to purify sporozoites from salivary gland debris before loading them onto a $10 \mathrm{x}$ Genomics' controller to generate single-cell RNA libraries. Both collection, purification, and capture workflows involve 1-3 hr of time from dissection to RNA capture, during which time the 
buffers and temperature used could impact sporozoite activation state (Hegge et al., 2010; Roth et al., 2018a). Additionally, for both the $P$. vivax and $P$. falciparum datasets, pseudotime analysis was found useful for understanding the developmental state of sporozoites, meaning the day, and possibly hour, of dissection post blood-meal could impact comparisons. Despite these differences, we note the substantial overlap in $P$. vivax and $P$. falciparum developmental states in low dimensional space. Still, for future studies these parameters should be standardized as much as possible to remove potential technical artefacts from comparisons.

Previous bulk sequencing analyses comparing $P$. vivax sporozoites and blood-stage parasites have revealed distinct transcription patterns between stages (Bozdech et al., 2008; Muller et al., 2019; Zhu et al., 2016). Here, we corroborate these findings and show that this variation is detectable using scRNA-seq technology. Our comparison of $P$. vivax sporozoites and blood stages highlights differences in transcription that may provide insights into the factors that allow the parasite to persist in different environments. Furthermore, our integrated analysis sets the stage for future additions, with the objective of generating a comprehensive $P$. vivax single-cell atlas. Our data serves as a new resource for the malaria research community, providing a detailed assessment of $P$. vivax gene usage in sporozoites at single-cell resolution. We anticipate that the dataset will serve as a launchpad to address other gaps in knowledge linked to $P$. vivax biology, including the extent to which various strains differ at the transcriptome level and how gene usage in individual sporozoites compares to other stages in the parasite's life cycle.

\section{Materials and methods}

\section{Ethics statement}

Research procedures and protocols for obtaining blood from patients were reviewed and approved by the Cambodian National Ethics Committee for Health Research (approval number: \#113NECHR). Protocols conformed to the World Medical Association Helsinki Declaration on Ethical Principles for Medical Research Involving Human Subjects (version 2002). Informed written consent was obtained from all volunteers or legal guardians.

\section{Blood samples, mosquitos, and infections}

Blood samples from symptomatic patients infected with $P$. vivax were collected at local health facilities in Mondulkiri province (Kaev Seima) in Eastern Cambodia from September to 
November 2019 (Figure 1A). Following a P. vivax gametocyte-containing blood meal, Anopheles dirus mosquitoes were maintained at $26^{\circ} \mathrm{C}$ on a $12 \mathrm{hr}: 12 \mathrm{hr}$ light:dark cycle and fed with $10 \%$ sucrose $+0.05 \%$ PABA solution. An. dirus found positive for $P$. vivax oocysts at six days post-feeding were transported to the Institut Pasteur of Cambodia Insectary Facility in Phnom Penh, Cambodia, where they were maintained under the same conditions described above.

\section{Sporozoite isolations}

$P$. vivax sporozoites were isolated from the salivary glands of female An. dirus mosquitoes 16-18 days after an infectious blood-meal. A team of technicians performed dissections for a maximum period of one hour. Generally, 75 to 100 mosquitoes were dissected in each one-hour sitting. Dissections were performed under a stereomicroscope, and salivary glands were placed in a microcentrifuge tube containing ice-cold Hanks Balanced Salt Solution. Sporozoites were then released from the salivary glands via manual disruption using a microcentrifuge pestle, and immediately purified using a discontinuous density gradient protocol adapted from Kennedy and colleagues (Kennedy et al., 2012), and as described previously (Ruberto et al., 2021). After purification, sporozoite mixtures were diluted in HBSS to 1000 sporozoites $/ \mu \mathrm{L}$ and were held on ice until further processing.

\section{Hepatocyte infections and liver-stage assessment}

$P$. vivax hepatocyte infections were performed as previously described (Roth et al., 2018b). Briefly, primary human hepatocytes (BiolVT) were seeded 2-3 days prior to infection with 15,000-20,000 sporozoites per well. Media was exchanged with fresh CP media (BiolVT) containing antibiotics the day after infection and every 2-3 days thereafter. At 12 days postinfection, cultures were fixed with $4 \%$ paraformaldehyde in 1X PBS. Fixed cultures were stained overnight at $4^{\circ} \mathrm{C}$ with recombinant mouse anti- $P$. vivax UIS4 antibody diluted $1: 25,000$ in a stain buffer (0.03\% TritonX-100 and 1\% (w/v) BSA in 1X PBS) (Schafer et al., 2018). Cultures were then washed thrice with $1 \mathrm{X}$ PBS and then stained overnight at $4^{\circ} \mathrm{C}$ with $1: 1000$ rabbit antimouse AlexaFluor TM488-conjugated antibody diluted 1:1,000 in stain buffer. Cultures were then washed thrice with 1X PBS and counterstained with $1 \mathrm{mg} / \mathrm{mL}$ Hoechst 33342 to detect parasite and host cell nuclear DNA. Infected hepatocytes were imaged on a Lionheart (Biotek). software (Biotek). 


\section{Single-cell partitioning, library preparation, and sequencing}

Approximately 5000-8000 sporozoites were loaded on a version 3- specific Chromium Chip. Chips containing sporozoite suspensions, Gel Beads in Emulsion (GEMs), and reverse transcription reagents were placed in a Chromium controller for single-cell partitioning and cellular barcoding. Barcoded cDNA libraries were generated according to the v3 Chromium Single Cell 3' gene expression protocol. cDNA libraries were loaded on individual flow cell lanes and sequenced using a HiSeq X Ten platform (Illumina) at Macrogen (Seoul, Korea). See Table S1 for sequencing statistics.

\section{Read alignment, cellular barcode assignment, and quantification}

The $P$. vivax P01 genome (version 3, October 2020) and its corresponding general feature format (gff) file (which contained UTR coordinates) were downloaded from ftp://ftp.sanger.ac.uk/pub/genedb/releases/latest/PvivaxP01 and used to create a genome index with STAR (v2.7.3a) (Dobin et al., 2013; Kaminow et al., 2021) with options: --runMode genomeGenerate -genomeSAindexNbases 11 -sjdbOverhang 90 (for v3 libraries), or 74 (for libraries derived from (Sà et al., 2020)). Mapping, demultiplexing and gene quantification was performed with STARsolo and the following options specified: --soloType CB_UMI_Simple -soloCBlen 16 --soloUMllen 12 (for v3 libraries) or 10 (for v2 libraries) --soloCBwhitelist /path/to/10x/version/specific/whitelist --alignIntronMin 1 --alignIntronMax 2756 --soloUMlfiltering MultiGeneUMI --soloFeatures Gene.

The An. dirus WRAIR2 genome and its corresponding GFF were downloaded from VectorBase (v49). We generated a genome index in STAR (v2.7.3a) with the same parameters as for the $P$. vivax P01 genome.

For a schematic of the alignment strategies, refer to Figure S2A and Figure S2C. A summary of read alignment statistics for each sample with- and without- the UTR information are found in Tables S2 and S12 for the sporozoite and blood-stage data, respectively.

\section{Filtering and normalization of scRNA-seq count matrices}

The unfiltered (raw) matrix, features, and barcodes files generated from STAR were imported into $\mathrm{R}$ (version 4) (R Core Team, 2013). We first removed rRNA encoding genes and then used emptyDrops function to distinguish between droplets containing cells and those only 
with ambient RNA to be discarded (Lun et al., 2019) with a lower library size limit of 40 to account for the low mRNA amounts in sporozoites (FDR < 0.001). We next removed cells with less than 60 unique genes detected in each cell library. Last, we filtered out lowly detected genes, retaining genes detected in at least two cells with more than two unique molecular identifier (UMI) counts. Post-cell and gene filtering, the data from each replicate were normalized using Seurat's 'LogNormalize' function with the default parameters selected (Stuart et al., 2019).

\section{Integration of $\boldsymbol{P}$. vivax sporozoite scRNA-seq data}

Filtered, normalized matrices from the three replicates were merged in a manner described in the Seurat (version 4) vignette, Introduction to scRNA-seq integration, available on the Satija Lab's website (https://satijalab.org/seurat/articles/integration introduction.html). Briefly, highly variable features were identified in each replicate using the 'FindVariableFeatures' function with the following parameters selected: selection method = "vst", features = (genes \# of detected in dataset) * 0.3 . Next, integration anchors were identified using the 'FindIntegrationAnchors' function with its default settings. Lastly, using these anchors, the three datasets were integrated using the 'IntegrateData' function with its default settings.

\section{Dimension reduction, clustering, and cluster marker identification.}

Post integration, data were scaled, and dimension reduction was performed using principal component analysis (PCA) to visualise the data in low-dimensional space. Next, the UMAP dimension reduction was performed using the RunUMAP function with the parameters dims $=1: 30$ and umap. method = "umap-learn", n.neighbours $=20$ and min.dist $=0.5$. Next, an unsupervised graph-based clustering approach was used to predict cell communities. First, knearest neighbours were identified, and a shared nearest neighbour graph was constructed using the FindNeighbours function under the default settings. Cell communities (clusters) were then identified using the FindClusters function with the Leiden algorithm (algorithm $=4$ ) selected. Clustering was performed at various resolutions (resolution $=0.1: 1$ ), and cluster stabilities were assessed using a Clustering tree plot (Zappia and Oshlack, 2018).

To detect cluster-specific markers (differentially transcribed genes), the Seurat function FinalAllMarkers was used with the following parameters: test.use $=$ "wilcox", $\min$. pct $=0.3$, 
496

497

min.diff.pct $=0.1$, only.pos $=$ TRUE, assay $=$ "originalexp". Differentially transcribed genes were considered significant if the adjusted $p$ value was below 0.05 .

\section{Trajectory analysis}

To infer the developmental trajectory of sporozoites, we used the Slingshot package (Street et al., 2018) to uncover the global structure of clusters of cells and convert this structure into a smoothed lineage representing "pseudotime". Lineages were first generated using the getLineages function on the UMAP embeddings generated previously (described in Dimension reduction, clustering and cluster marker identification). Cluster C1 was selected as the starting cluster because it contained putative immature salivary gland sporozoite markers. Next, smoothed lineage curves were constructed using the getCurves function with the default parameters. We then used the tradeSeq package (van den Berge et al., 2020) to analyze transcription along the trajectory. To this end, we ran the fitGAM function on the SlingshotDataset to fit a negative binomial general additive model on the data. Based on the fitted models, the AssociationTest with default settings selected was used to test transcription changes across pseudotime. Genes were considered significantly associated with a change over pseudotime at a false discovery rate below 0.01 .

\section{Inter-species comparison of Plasmodium sporozoites}

We grouped orthologous genes of $P$. vivax P01, P. cynomolgi $M, P$. cynomolgi $B, P$. berghei ANKA, P. falciparum 3D7, $P$. yoelli yoelli 17X, $P$. chabaudi chabaudi, $P$. knowlesi $H, P$. malariae UG01, P. ovale curtisi GH01 and Toxoplasma gondii ME49 using OrthoFinder (Emms and Kelly, 2019). Parameters were kept at default and gene fasta files for input were obtained from either PlasmoDB or ToxoDB, release version 51. From the 'Orthogroups' analysis, the tabdelimited output file was used to extract the species-specific gene IDs for $P$. vivax and $P$. falciparum and match these with a universal orthogroup ID (Table S7). We used this orthogroup ID to replace rownames corresponding to $P$. vivax or $P$. falciparum gene IDs in the count matrices of $P$. vivax sporozoites (this study) and $P$. falciparum sporozoites (Real et al., 2021). Orthogroup IDs for merge were only retained if they had only one entry per species. In a $P$. vivax-centric approach, we retained orthogroup IDs if either $P$. falciparum, $P$. berghei ANKA, $P$. cynomolgi $M$ or $P$. cynomolgi $\mathrm{B}$ had at least one corresponding gene orthologue. $P$. vivax data were processed as described earlier (section Filtering and normalization of scRNA-seq count matrices) and $P$. falciparum data was obtained from 
https://github.com/vhowick/pf moz stage atlas/tree/master/counts and metadata. Following

528

529

530

531

532

533

534

535

536

537

538

539

540

541

542

543

544

545

546

547

548

549

550

551

552

553

554

555

556

557

558

the replacement of gene IDs with new orthogroup IDs, we additionally performed another round of cell filtering to account for changes in gene count per cell information with the removal of some genes without equivalent orthogroup IDs. Data integration were also performed as described earlier (section Integration of $P$. vivax sporozoite scRNA-seq datasets). The $P$. vivax data were randomly subset (500 sporozoites per replicate) for subsequent analyses to match the proportion of cells in the $P$. falciparum dataset prior to integration and account for the disproportionate number of sporozoites between the two species. Clusters of sporozoites were identified using 'FindNeighbours' function using the 'pca' reduction, dims $=1: 15$, k. param $=20$, and 'FindClusters' function with the Leiden clustering algorithm (algorithm $=4$ ) at a resolution of 0.2. Marker genes for the defined clusters were identified with either the 'FinderConservedMarkers' or 'FindMarkers' functions (Seurat) using a Wilcoxon Rank Sum test. The number of cells, percent difference, and fold-change parameters for each of the analyses are indicated in the $\mathrm{R}$ markdown document provided.

\section{Integration of $P$. vivax sporozoite and blood stage scRNA-seq data}

Filtering and normalization for the $P$. vivax blood-stage scRNA-seq data was performed in the same manner used for the sporozoite scRNA-seq data described in Filtering and normalization of scRNA-seq count matrices. Of the $10 P$. vivax blood-stage replicates, we used the 7 generated from samples without chloroquine treatment. Before integration, variable features for the combined sporozoite (3) and combined blood-stage (7) datasets were identified using the FindVariableFeatures function with the following parameters: selection method = "vst", features $=($ genes $\#$ of detected in dataset $){ }^{*} 0.3$. Next, we used the FindIntegrationAnchors function to identify anchors between the sporozoite and blood-stage datasets. Guided by the recommendations provided by the Satija lab's vignette Fast integration using reciprocal PCA (RPCA) (https://satijalab.org/seurat/articles/integration rpca.html), namely when cells in one dataset have no matching type in the other, we selected the RPCA parameter in FindIntegrationAnchors (reduction = "rpca") to identify anchors between the sporozoite and blood-stage data. Last, using these anchors, the datasets were integrated using the IntegrateData function with its default settings. Assessment of differentially transcribed genes in sporozoite and blood-stage parasites were performed in the same manner described in Differential genes transcription analysis with additional parameters indicated in the provided $\mathrm{R}$ markdown files. 


\section{Contact for reagent and resource sharing}

560 Further information and requests for resources and reagents should be directed to the Lead

561 Contact, Ivo Mueller (e-mail: mueller@wehi.edu.au).

\section{Data Availability:}

All raw sequencing data generated from $P$. vivax sporozoites in this study will be deposited and

564 accessible in the European Nucleotide Archive (www.ebi.ac.uk/ena/) upon acceptance of the

565 manuscript.

566 P. vivax blood-stage scRNA-seq data were downloaded from NCBl's Short Read Archive

567 (Bioproject ID: PRJNA603327).

$P$. falciparum sporozoite scRNA-seq data were obtained from:

569 https://github.com/vhowick/pf_moz_stage_atlas

570 Scripts and supporting files will be available on GitHub upon acceptance of the manuscript.

571 Archived scripts and output files as at time of publication will be available on Zenodo.com upon

572 acceptance of the manuscript.

\section{Supporting information}

\section{Abbreviations}

575 FDR: false discovery rate, GEM: Gel Beads in Emulsion, GFF: General Feature Format, mRNA:

576 messenger RNA, PBS: phosphate-buffered saline, PCA: principal components analysis, rRNA:

577 ribosomal RNA, scRNA-seq: single-cell RNA sequencing, UMAP: uniform manifold

578 approximation projections, UIS4: Upregulated in-infective sporozoite 4, UMIs: unique molecular 579 identifiers, UTRs: untranslated regions.

\section{Acknowledgments}

582 We thank the P. vivax patients of Mondulkiri Province, Cambodia, for participating in this study.

583 We thank the Institut Pasteur insectary staff (Makara Pring, Koeun Kaing, Nora Sambath) for 
584 An. dirus mosquito colony maintenance, the laboratory staff (Eakpor Piv, Chansophea Chhin,

585 Sreyvouch Phen, Chansovandan Chhun, Sivcheng Phal, Baura Tat) for assistance with the

586 mosquito dissections and the in vitro assays, and the field site manager (Saorin Kim) for

587 logistical assistance. This work was supported by the Agence Nationale de la Recherche (ANR-

588 17-CE13-0025 to A.A.R, G.S., I.M.), the National Health and Medical Research Council of

589 Australia (NHMRC; 1092789 and 1134989 to I.M.), and a NHMRC Principal Research

590 Fellowship (1155075 to I.M).

591 Author Contributions

592 Conceptualization: Anthony Ruberto, Caitlin Bourke, Amélie Vantaux, Aaron Jex, Benoit

593 Witkowski, Georges Snounou, Ivo Mueller

594 Formal analysis: Anthony Ruberto, Caitlin Bourke

595 Funding acquisition: Georges Snounou, Ivo Mueller

596 Investigation: Anthony Ruberto, Caitlin Bourke, Steven P. Maher, Amélie Vantaux

597 Methodology: Anthony Ruberto, Caitlin Bourke, Steven P. Maher, Amélie Vantaux, Benoit

598 Witkowski, Georges Snounou, Ivo Mueller

599 Project administration: Ivo Mueller

600 Supervision: Aaron Jex, Ivo Mueller

601 Visualization: Anthony Ruberto, Caitlin Bourke

602 Writing - original draft: Anthony Ruberto, Caitlin Bourke

603 Writing - review \& editing: Anthony Ruberto, Caitlin Bourke, Steven P. Maher, Amélie

604 Vantaux, Aaron Jex, Benoit Witkowski, Georges Snounou, Ivo Mueller

605 Competing interests

606 The authors declare no competing interests.

607 


\section{References}

609 Adekunle, A.I., Pinkevych, M., McGready, R., Luxemburger, C., White, L.J., Nosten, F., Cromer, D., and Davenport, M.P. (2015). Modeling the Dynamics of Plasmodium vivax Infection and Hypnozoite

611 Reactivation In Vivo. PLOS Neglected Tropical Diseases 9, e0003595.

612 Al-Nihmi, F.M.A., Kolli, S.K., Reddy, S.R., Mastan, B.S., Togiri, J., Maruthi, M., Gupta, R., Sijwali, P.S., 613 Mishra, S., and Kumar, K.A. (2017). A Novel and Conserved Plasmodium Sporozoite Membrane Protein 614 SPELD is Required for Maturation of Exo-erythrocytic Forms. Scientific Reports 7.

615 Amino, R., Thiberge, S., Martin, B., Celli, S., Shorte, S., Frischknecht, F., and Ménard, R. (2006).

616 Quantitative imaging of Plasmodium transmission from mosquito to mammal. Nature Medicine 12, 220617224.

618 Battle, K.E., and Baird, K.J. (2021). The global burden of Plasmodium vivax malaria is obscure and 619 insidious. PLOS Medicine 18, e1003799.

620 Battle, K.E., Karhunen, M.S., Bhatt, S., Gething, P.W., Howes, R.E., Golding, N., van Boeckel, T.P., 621 Messina, J.P., Shanks, G.D., Smith, D.L., et al. (2014). Geographical variation in Plasmodium vivax relapse. 622 Malaria Journal 13.

623 Battle, K.E., Lucas, T.C.D., Nguyen, M., Howes, R.E., Nandi, A.K., Twohig, K.A., Pfeffer, D.A., Cameron, E., 624 Rao, P.C., Casey, D., et al. (2019). Mapping the global endemicity and clinical burden of Plasmodium 625 vivax, 2000-17: a spatial and temporal modelling study. The Lancet 394, 332-343. van den Berge, K., Roux de Bézieux, H., Street, K., Saelens, W., Cannoodt, R., Saeys, Y., Dudoit, S., and Clement, L. (2020). Trajectory-based differential expression analysis for single-cell sequencing data.

628 Nature Communications 11.

629 Bogale, H.N., Pascini, T. v., Kanatani, S., Sá, J.M., Wellems, T.E., Sinnis, P., Vega-Rodríguez, J., and Serre, 630 D. (2021). Transcriptional heterogeneity and tightly regulated changes in gene expression during 631 Plasmodium berghei sporozoite development. Proceedings of the National Academy of Sciences of the 632 United States of America 118.

633 Bozdech, Z., Mok, S., Hu, G., Imwong, M., Jaidee, A., Russell, B., Ginsburg, H., Nosten, F., Day, N.P.J., 634 White, N.J., et al. (2008). The transcriptome of Plasmodium vivax reveals divergence and diversity of 635 transcriptional regulation in malaria parasites. Proceedings of the National Academy of Sciences of the 636 United States of America 105, 16290-16295.

637 Briquet, S., Marinach, C., Silvie, O., and Vaquero, C. (2021). Preparing for Transmission: Gene Regulation 638 in Plasmodium Sporozoites. Frontiers in Cellular and Infection Microbiology 10, 907.

639 Cerami, C., Frevert, U., Sinnis, P., Takacs, B., Clavijo, P., Santos, M.J., and Nussenzweig, V. (1992). The 640 basolateral domain of the hepatocyte plasma membrane bears receptors for the circumsporozoite 641 protein of plasmodium falciparum sporozoites. Cell 70.

642 Commons, R.J., Simpson, J.A., Watson, J., White, N.J., and Price, R.N. (2020). Estimating the Proportion 643 of Plasmodium vivax Recurrences Caused by Relapse: A Systematic Review and Meta-Analysis. American 644 Journal of Tropical Medicine and Hygiene 103, 1094-1099. 
645

646

647

648

649

650

651

652

653

654

655

656

657

658

659

660

661

662

663

664

665

666

667

668

669

670

671

672

673

674

675

676

677

678

679

680

Coppi, A., Natarajan, R., Pradel, G., Bennett, B.L., James, E.R., Roggero, M.A., Corradin, G., Persson, C., Tewari, R., and Sinnis, P. (2011). The malaria circumsporozoite protein has two functional domains, each with distinct roles as sporozoites journey from mosquito to mammalian host. Journal of Experimental Medicine 208.

Dobin, A., Davis, C.A., Schlesinger, F., Drenkow, J., Zaleski, C., Jha, S., Batut, P., Chaisson, M., and Gingeras, T.R. (2013). STAR: Ultrafast universal RNA-seq aligner. Bioinformatics 29, 15-21.

Emms, D.M., and Kelly, S. (2019). OrthoFinder: Phylogenetic orthology inference for comparative genomics. Genome Biology 20, 238.

Engelmann, S., Silvie, O., and Matuschewski, K. (2009). Disruption of Plasmodium sporozoite transmission by depletion of sporozoite invasion-associated protein 1. Eukaryotic Cell 8.

Gomes-Santos, C.S.S., Braks, J., Prudêncio, M., Carret, C., Gomes, A.R., Pain, A., Feltwell, T., Khan, S., Waters, A., Janse, C., et al. (2011). Transition of Plasmodium sporozoites into liver stage-like forms is regulated by the RNA binding protein Pumilio. PLoS Pathogens 7.

Haque, A., Engel, J., Teichmann, S.A., and Lönnberg, T. (2017). A practical guide to single-cell RNAsequencing for biomedical research and clinical applications. Genome Medicine 9, 1-12.

Hegge, S., Kudryashev, M., Barniol, L., and Frischknecht, F. (2010). Key factors regulating Plasmodium berghei sporozoite survival and transformation revealed by an automated visual assay. FASEB Journal : Official Publication of the Federation of American Societies for Experimental Biology 24, 5003-5012.

Heiss, K., Nie, H., Kumar, S., Daly, T.M., Bergman, L.W., Matuschewski, K., Matuschewski, K., and Heidelberg, : (2008). Functional Characterization of a Redundant Plasmodium TRAP Family Invasin, TRAP-Like Protein, by Aldolase Binding and a Genetic Complementation Test Mailing address for. EUKARYOTIC CELL 7, 1062-1070.

Howick, V.M., Russell, A.J.C., Andrews, T., Heaton, H., Reid, A.J., Natarajan, K., Butungi, H., Metcalf, T., Verzier, L.H., Rayner, J.C., et al. (2019). The Malaria Cell Atlas: Single parasite transcriptomes across the complete Plasmodium life cycle. Science 365, eaaw2619.

Huldén, L., Huldén, L., and Heliövaara, K. (2008). Natural relapses in vivax malaria induced by Anopheles mosquitoes. Malaria Journal 7.

Ishino, T., Yano, K., Chinzei, Y., and Yuda, M. (2004). Cell-passage activity is required for the malarial parasite to cross the liver sinusoidal cell layer. PLoS Biology 2.

Kaminow, B., Yunusov, D., and Dobin, A. (2021). STARsolo: accurate, fast and versatile mapping/quantification of single-cell and single-nucleus RNA-seq data. BioRxiv 2021.05.05.442755.

Kennedy, M., Fishbaugher, M.E., Vaughan, A.M., Patrapuvich, R., Boonhok, R., Yimamnuaychok, N., Rezakhani, N., Metzger, P., Ponpuak, M., Sattabongkot, J., et al. (2012). A rapid and scalable density gradient purification method for Plasmodium sporozoites. Malaria Journal 11, 421.

Kivioja, T., Vähärautio, A., Karlsson, K., Bonke, M., Enge, M., Linnarsson, S., and Taipale, J. (2011).

Counting absolute numbers of molecules using unique molecular identifiers. Nature Methods 9, 72-74. 
Klein, A.M., Mazutis, L., Akartuna, I., Tallapragada, N., Veres, A., Li, V., Peshkin, L., Weitz, D.A., and Kirschner, M.W. (2015). Droplet barcoding for single-cell transcriptomics applied to embryonic stem cells. Cell 161, 1187-1201.

Kong, J., and Lasko, P. (2012). Translational control in cellular and developmental processes. Nature Reviews Genetics 2012 13:6 13, 383-394.

Krotoski, W.A. (1985). Discovery of the hypnozoite and a new theory of malarial relapse. Transactions of the Royal Society of Tropical Medicine and Hygiene 79, 1.

Krotoski, W.A., Collins, W.E., Bray, R.S., Garnham, P.C., Cogswell, F.B., Gwadz, R.W., Killick-Kendrick, R., Wolf, R., Sinden, R., Koontz, L.C., et al. (1982). Demonstration of hypnozoites in sporozoite-transmitted Plasmodium vivax infection. The American Journal of Tropical Medicine and Hygiene 31, 1291-1293.

Lindner, S.E., Mikolajczak, S.A., Vaughan, A.M., Moon, W., Joyce, B.R., Sullivan, W.J., and Kappe, S.H.I. (2013). Perturbations of PlasmodiumPuf2 expression and RNA-seq of Puf2-deficient sporozoites reveal a critical role in maintaining RNA homeostasis and parasite transmissibility. Cellular Microbiology 15, 1266-1283.

Lun, A.T.L., Riesenfeld, S., Andrews, T., Dao, T.P., Gomes, T., and Marioni, J.C. (2019). EmptyDrops: Distinguishing cells from empty droplets in droplet-based single-cell RNA sequencing data. Genome Biology 20, 63.

Lysenko, A.J., Beljaev, A.E., and Rybalka, V.M. (1977). Population studies of Plasmodium vivax. 1. The theory of polymorphism of sporozoites and epidemiological phenomena of tertian malaria.

Macosko, E.Z., Basu, A., Satija, R., Nemesh, J., Shekhar, K., Goldman, M., Tirosh, I., Bialas, A.R., Kamitaki, N., Martersteck, E.M., et al. (2015). Highly parallel genome-wide expression profiling of individual cells using nanoliter droplets. Cell 161, 1202-1214.

Matuschewski, K., Ross, J., Brown, S.M., Kaiser, K., Nussenzweig, V., and Kappe, S.H.I. (2002). Infectivityassociated Changes in the Transcriptional Repertoire of the Malaria Parasite Sporozoite Stage *. Journal of Biological Chemistry 277, 41948-41953.

Mikolajczak, S.A., Vaughan, A.M., Kangwanrangsan, N., Roobsoong, W., Fishbaugher, M., Yimamnuaychok, N., Rezakhani, N., Lakshmanan, V., Singh, N., Kaushansky, A., et al. (2015). Plasmodium vivax liver stage development and hypnozoite persistence in human liver-chimeric mice. Cell Host and Microbe 17, 526-535.

Mueller, A.K., Camargo, N., Kaiser, K., Andorfer, C., Frevert, U., Matuschewski, K., and Kappe, S.H.I. (2005). Plasmodium liver stage developmental arrest by depletion of a protein at the parasite-host interface. Proceedings of the National Academy of Sciences 102, 3022-3027.

Muller, I., Jex, A.R., Kappe, S.H.I., Mikolajczak, S.A., Sattabongkot, J., Patrapuvich, R., Lindner, S., Flannery, E.L., Koepfli, C., Ansell, B., et al. (2019). Transcriptome and histone epigenome of Plasmodium vivax salivary-gland sporozoites point to tight regulatory control and mechanisms for liver-stage differentiation in relapsing malaria. International Journal for Parasitology 49, 501-513. 
Packer, J.S., Zhu, Q., Huynh, C., Sivaramakrishnan, P., Preston, E., Dueck, H., Stefanik, D., Tan, K., Trapnell, C., Kim, J., et al. (2019). A lineage-resolved molecular atlas of C. Elegans embryogenesis at single-cell resolution. Science 365.

720 R Core Team (2013). R: A Language and Environment for Statistical Computing.

721 Real, E., Rodrigues, L., Cabal, G.G., Enguita, F.J., Mancio-Silva, L., Mello-Vieira, J., Beatty, W., Vera, I.M., 722 Zuzarte-Luís, V., Figueira, T.N., et al. (2017). Plasmodium UIS3 sequesters host LC3 to avoid elimination 723 by autophagy in hepatocytes. Nature Microbiology 2017 3:1 3, 17-25.

724 Real, E., Howick, V.M., Dahalan, F.A., Witmer, K., Cudini, J., Andradi-Brown, C., Blight, J., Davidson, M.S., 725 Dogga, S.K., Reid, A.J., et al. (2021). A single-cell atlas of Plasmodium falciparum transmission through 726 the mosquito. Nature Communications 12, 3196.

727 Robinson, L.J., Wampfler, R., Betuela, I., Karl, S., White, M.T., Li Wai Suen, C.S.N., Hofmann, N.E., 728 Kinboro, B., Waltmann, A., Brewster, J., et al. (2015). Strategies for Understanding and Reducing the 729 Plasmodium vivax and Plasmodium ovale Hypnozoite Reservoir in Papua New Guinean Children: A 730 Randomised Placebo-Controlled Trial and Mathematical Model. PLOS Medicine 12, e1001891. Roth, A., Adapa, S.R., Zhang, M., Liao, X., Saxena, V., Goffe, R., Li, S., Ubalee, R., Saggu, G.S., Pala, Z.R., et al. (2018a). Unraveling the Plasmodium vivax sporozoite transcriptional journey from mosquito vector to human host. Scientific Reports 8, 12183.

Roth, A., Maher, S.P., Conway, A.J., Ubalee, R., Chaumeau, V., Andolina, C., Kaba, S.A., Vantaux, A., Bakowski, M.A., Thomson-Luque, R., et al. (2018b). A comprehensive model for assessment of liver stage therapies targeting Plasmodium vivax and Plasmodium falciparum. Nature Communications 9, 1837. Ruberto, A.A., Bourke, C., Merienne, N., Obadia, T., Amino, R., and Mueller, I. (2021). Single-cell RNA sequencing reveals developmental heterogeneity among Plasmodium berghei sporozoites. Scientific Reports 11, 4127.

Sà, J.M., Cannon, M. v., Caleon, R.L., Wellems, T.E., and Serre, D. (2020). Single-cell transcription analysis of Plasmodium vivax blood-stage parasites identifies stage- And species-specific profiles of expression. PLoS Biology 18, e3000711.

Sattabongkot, J., Suansomjit, C., Nguitragool, W., Sirichaisinthop, J., Warit, S., Tiensuwan, M., and Buates, S. (2018). Prevalence of asymptomatic Plasmodium infections with sub-microscopic parasite densities in the northwestern border of Thailand: A potential threat to malaria elimination. Malaria

746 Journal 17.

747 Schafer, C., Dambrauskas, N., Steel, R.W., Carbonetti, S., Chuenchob, V., Flannery, E.L., Vigdorovich, V., 748 Oliver, B.G., Roobsoong, W., Maher, S.P., et al. (2018). A recombinant antibody against Plasmodium 749 vivax UIS4 for distinguishing replicating from dormant liver stages. Malaria Journal 17, 370.

750 Schäfer, C., Zanghi, G., Vaughan, A.M., and Kappe, S.H.I. (2021). Plasmodium vivax Latent Liver Stage 751 Infection and Relapse: Biological Insights and New Experimental Tools. Annual Review of Microbiology 75275. 
Shields, E.J., Sorida, M., Sheng, L., Sieriebriennikov, B., Ding, L., and Bonasio, R. (2021). Genome annotation with long RNA reads reveals new patterns of gene expression in an ant brain. BioRxiv 2021.04.20.440671.

Siegel, S. v., Chappell, L., Hostetler, J.B., Amaratunga, C., Suon, S., Böhme, U., Berriman, M., Fairhurst, R.M., and Rayner, J.C. (2020). Analysis of Plasmodium vivax schizont transcriptomes from field isolates reveals heterogeneity of expression of genes involved in host-parasite interactions. Scientific Reports 10, 16667.

Sinnis, P., Clavijo, P., Fenyö, D., Chait, B.T., Cerami, C., and Nussenzweig, V. (1994). Structural and functional properties of region II-plus of the malaria circumsporozoite protein. Journal of Experimental Medicine 180.

Street, K., Risso, D., Fletcher, R.B., Das, D., Ngai, J., Yosef, N., Purdom, E., and Dudoit, S. (2018). Slingshot: Cell lineage and pseudotime inference for single-cell transcriptomics. BMC Genomics 19.

Stuart, T., Butler, A., Hoffman, P., Hafemeister, C., Papalexi, E., Mauck, W.M., Hao, Y., Stoeckius, M., Smibert, P., and Satija, R. (2019). Comprehensive Integration of Single-Cell Data. Cell 177, 18881902.e21.

Talman, A.M., Lacroix, C., Marques, S.R., Blagborough, A.M., Carzaniga, R., Ménard, R., and Sinden, R.E. (2011). PbGEST mediates malaria transmission to both mosquito and vertebrate host. Molecular Microbiology 82.

Traag, V.A., Waltman, L., and van Eck, N.J. (2019). From Louvain to Leiden: guaranteeing well-connected communities. Scientific Reports 9, 1-12.

Ungureanu, E., Killick-Kendrick, R., Garnham, P.C.C., Branzei, P., Romanescu, C., and Shute, P.G. (1976). Prepatent periods of a tropical strain of Plasmodium vivax after inoculations of tenfold dilutions of sporozoites. Transactions of the Royal Society of Tropical Medicine and Hygiene 70, 482-483.

Weiss, D.J., Lucas, T.C.D., Nguyen, M., Nandi, A.K., Bisanzio, D., Battle, K.E., Cameron, E., Twohig, K.A., Pfeffer, D.A., Rozier, J.A., et al. (2019). Mapping the global prevalence, incidence, and mortality of Plasmodium falciparum, 2000-17: a spatial and temporal modelling study. The Lancet 394, 322-331.

Westenberger, S.J., McClean, C.M., Chattopadhyay, R., Dharia, N. v., Carlton, J.M., Barnwell, J.W., Collins, W.E., Hoffman, S.L., Zhou, Y., Vinetz, J.M., et al. (2010). A Systems-Based Analysis of Plasmodium vivax Lifecycle Transcription from Human to Mosquito. PLoS Neglected Tropical Diseases 4, e653.

White, N.J. (2011). Determinants of relapse periodicity in Plasmodium vivax malaria. Malaria Journal 10. White, M.T., Walker, P., Karl, S., Hetzel, M.W., Freeman, T., Waltmann, A., Laman, M., Robinson, L.J., Ghani, A., and Mueller, I. (2018). Mathematical modelling of the impact of expanding levels of malaria control interventions on Plasmodium vivax. Nature Communications 2018 9:1 9, 1-10.

World Health Organization (2020). World malaria report 2020.

Zappia, L., and Oshlack, A. (2018). Clustering trees: a visualization for evaluating clusterings at multiple resolutions. GigaScience 7. 
789 Zhang, B., Gallegos, M., Puoti, A., Durkin, E., Fields, S., Kimble, J., and Wickens, M.P. (1997). A conserved 790 RNA-binding protein that regulates sexual fates in the C. elegans hermaphrodite germ line. Nature 1997 $791390: 6659390,477-484$.

792 Zheng, G.X.Y., Terry, J.M., Belgrader, P., Ryvkin, P., Bent, Z.W., Wilson, R., Ziraldo, S.B., Wheeler, T.D., 793 McDermott, G.P., Zhu, J., et al. (2017). Massively parallel digital transcriptional profiling of single cells. $794 \quad$ Nature Communications 8.

795 Zhu, L., Mok, S., Imwong, M., Jaidee, A., Russell, B., Nosten, F., Day, N.P., White, N.J., Preiser, P.R., and 796 Bozdech, Z. (2016). New insights into the Plasmodium vivax transcriptome using RNA-Seq. Scientific 797 Reports 6. 